\title{
Use of a bounding surface model in predicting element tests and capacity in boundary value problems
}

\author{
A. Roy' ${ }^{1}$, S. H. Chow ${ }^{2}$, C. D. O'Loughlin ${ }^{3}$, M.F. Randolph ${ }^{4}$, S. Whyte ${ }^{5}$ \\ ${ }^{1}$ Corresponding Author \\ PhD student, Centre for Offshore Foundation Systems (BEng, MEng) \\ The University of Western Australia \\ 35 Stirling Highway, Perth WA 6009, Perth, Australia \\ Phone: +61 42225 9766, Fax: +61 864881044 \\ E-mail: anamitra.roy@research.uwa.edu.au \\ ${ }^{2}$ Senior Lecturer, Department of Infrastructure Engineering (BEng, MEng, PhD) \\ Engineering Block B, Grattan Street, Parkville \\ The University of Melbourne, Victoria 3010, Australia \\ Phone: +61 390357504 \\ E-mail: shiaohuey.chow@unimelb.edu.au \\ ${ }^{3}$ Associate Professor, Centre for Offshore Foundation Systems (BEng, PhD) \\ The University of Western Australia \\ 35 Stirling Highway, Perth WA 6009, Perth, Australia \\ Phone: +6186488 6488, Fax: +61 864881044 \\ E-mail: conleth.oloughlin@uwa.edu.au \\ ${ }^{4}$ Professor, Centre for Offshore Foundation Systems (MA, PhD, FAA, FREng, FRS, FTSE, FIEAust, CPEng) \\ The University of Western Australia \\ 35 Stirling Highway, Perth WA 6009, Perth, Australia \\ Phone: +61 86488 3075, Fax: +61 864881044 \\ E-mail: mark.randolph@uwa.edu.au \\ ${ }^{5}$ Former DEng Candidate, Department of Engineering Science (BEng, MSc) \\ University of Oxford \\ Phone: +44 (0) 1491820870 \\ Email: scott.whyte@eng.ox.ac.uk
}

Number of words $\quad 8000$ (excluding abstract, references and captions)

Number of figures $\quad 19$

Number of tables $\quad 4$ 


\begin{abstract}
The paper examines the merit of a bounding surface plasticity model through an optimised calibration procedure and assesses the model performance in capturing the response at both element and system level. The governing equations are based essentially on the parent bounding surface plasticity model developed by Dafalias and Manzari (2004) with some simple yet practical changes to enable realistic predictions for monotonic loading along different load paths. This is achieved by scaling the influence of state parameter based on a normalised measure of anisotropy, thus leading to suitable change in dilatancy and plastic modulus for different loading directions. The paper presents a simple optimisation technique for calibrating the model parameters, providing an objective approach to reduce the uncertainties in parameter determination that leads to good agreement with responses measured in drained and undrained triaxial tests. The model has also been implemented for the boundary value problem of a buried circular plate anchor and a surface circular footing. Comparisons of the simulated responses with those measured in centrifuge tests demonstrate the potential of the model, whilst also pointing to the challenges in capturing the global response at all strain levels, even for rather simple boundary value problems.
\end{abstract}

\title{
Keywords
}

Bounding surface plasticity model; non-associated plasticity; model calibration; sands; circular footings 


\section{Introduction}

Successful application of numerical simulations in geotechnical boundary value problems relies on the appropriateness of the soil constitutive model employed within the numerical analysis. In geotechnical problems, plasticity bound theorems (Drucker and Prager 1952) or finite element (FE) analysis with non-linear elastic models (Duncan and Chang 1970) and elastic-perfectly plastic models (Loukidis et al. 2008; Tom et al. 2017) have been used extensively due to their simplicity, minimal requirements for complex parameter determination and their availability in libraries of commercial FE codes. Many of these models are based on associated plasticity and are incapable of reproducing the post peak softening response exhibited by coarse grained soils. Hence, the stress-strain response captured at element level by such models is inherently oversimplified, resulting in less confident predictions for boundary value problems.

The shortcomings of the more routine soil models have been addressed through the introduction of new concepts, such as multi-surface plasticity (Yang et al. 2003), hypoplasticity (Gudehus 1996) and bounding surface plasticity (Dafalias and Popov 1975) to existing constitutive models. Among these concepts, models with bounding surface plasticity having a critical state skeleton (Roscoe et al. 1958), have generated considerable interest, as they allow for more realistic stress-strain predictions (Gajo and Muir Wood 1999; Manzari and Dafalias 1997; Li and Dafalias 2002; Dafalias and Manzari 2004; Dafalias et al. 2004; Taiebat and Dafalias 2008; Loukidis and Salgado 2009; Taborda et al. 2014; Petalas et al. 2019). However, in spite of their impressive performance at element level in capturing the mechanical behaviour of coarse-grained soils, application of such models for real boundary value problems has been fairly limited; e.g. footing bearing capacity analysis (Loukidis and Salgado 2011; Chaloulos et al. 2019) and geotechnical earthquake engineering applications (Ziotopoulou and Boulanger 2013). The limited application of such models in geotechnical literature may be because the added flexibility of such models (which by extension allows for 'real' soil response to be predicted more accurately) comes at the cost of an increased number of model parameters, which then necessitates a broader range of element tests to optimise the parameters. As many of the parameters are unfamiliar to practitioners, there is often apprehension and reluctance to adopt the more advanced models with their non-trivial calibration process. In view of these barriers to uptake, this paper outlines a simple optimisation technique for parameter determination for the SANISAND model, demonstrating its application at both soil element level and in boundary value problems.

The paper first provides an overview of the adopted model and introduces some minor modifications relating to the treatment of fabric anisotropy. This is followed by the description of an optimisation routine for 
calibrating the model parameters, as an alternate and more systematic approach to parameter determination than simple trial and error approaches. The merit of the minor modifications in model features, coupled with the optimisation routine, is then examined by comparing measured and simulated responses of drained and undrained triaxial tests on Erksak sand and UWA silica sand. Finally, the definitive test of the model performance is a set of simulations (using the optimised parameters for UWA silica sand) of the monotonic response of a buried circular plate anchor and a surface circular footing in UWA silica sand.

\section{Key features of the constitutive model}

The SANISAND constitutive framework adopted here is the one presented in Dafalias and Manzari (2004) or Dafalias et al. (2004) since it embraces typical bounding surface plasticity features with use of least parameters, and is briefly summarised for completeness. The model controls sand behaviour with the simultaneous interaction of the yield surface with three other surfaces: critical state surface, bounding surface and dilatancy surface as shown in Fig. 1. The position of these surfaces depend on the current values of Lode angle $(\theta)$ and the state parameter ( $\psi$ ) (Eqs. (8) to (12) in Table 1). The governing equations are presented in Table 1 (Eqs. (1) to (26)).

Although versions of SANISAND with a closing cap have been developed (Taiebat and Dafalias 2008), the version used here has a yield surface is in the form of an open cone with its apex at the origin of stress space (Eq. (7) in Table 1); this serves to delineate the start of the plastic strain region. This version of the model is sufficient for medium to dense sand states. If the stresses are within the current yield surface, the soil behaves elastically with its behaviour being controlled solely by the elastic shear modulus $(G)$ and bulk modulus $(K)$, as evident in Eqs. (3) to (6) in Table 1. With pressure and density exerting a dominant control on $G$ and $K$ (Yamashita et al. 2000), these quantities in the model are stress level and density dependent. Owing to the open cone formulation, plastic shear and volume strains are simulated only with changes in deviatoric stress ratio $\left(d \eta=d\left(\frac{q}{p^{\prime}}\right)\right.$ $\neq 0$, where $q$ is the deviatoric stress and $p^{\prime}$ is the mean effective stress).

The updated soil state position for a given increment of strain is controlled by the plastic modulus $\left(K_{p}\right)$. Although there appears to be different adaptations for the expressions of $K_{p}$ within the SANISAND family of models (details in Li and Dafalias 2000; Taborda et al. 2014), the dominating feature is governed by the distance from the current stress state to the bounding surface. The model used here uses the $K_{p}$ expression from Dafalias et al. (2004) (Eqs. (13) to (15) in Table 1). Based on the value of $K_{p}$ (whether positive or negative), the model is able to predict hardening and softening behaviour. The magnitude of incremental plastic volumetric strains is 
influenced by the distance between the current stress point and its projected image on the dilatancy surface (Eq. (20) in Table 1). As the soil continues to deform plastically, the kinematic hardening nature of the model ensures that the yield surface rotates (causing its axis to shift) so as keep the current stress state on the surface. The dilatancy and bounding surfaces are expressed mathematically as exponential forms of $\psi$. This feature has a twofold advantage: firstly, it unifies the treatment of loose and dense sands under one constitutive framework and secondly, it allows the surfaces to collapse on to the critical state surface as $\psi$ approaches zero, thus making it possible to simulate stress and volume constancy at critical state (Been et al. 1991).

In the version of the model adopted here, different deviatoric functions (Eq. (19)) are used to control the shape of the critical state surface (denoted as $g_{1}\left(\theta, c_{1}\right)$ in Eq. (10)) and plastic potential $\left(g_{2}\left(\theta, c_{2}\right)\right.$ (different from $\left.g_{1}\right)$ ). This feature is consistent with the model version adopted by Loukidis and Salgado (2009). The mathematical form of $g_{1}\left(\theta, c_{1}\right)$ was based on Sheng et al. (2000), simply to retain convexity of the bounding surfaces for $c_{1}$ values as low as 0.61. As noted by Potts and Gens (1984) and Grammatikopoulou et al. (2007), $\theta$ at failure for a plane strain deformation problem is influenced only by the shape of the plastic potential function. In the current model, with the use of two different deviatoric functions, the value of $c_{l}$ no longer influences the plastic potential function or the values of $\theta$ and $b$ mobilized at critical state under non-triaxial loading conditions. This would be impossible to achieve with the use of a single deviatoric function.

Another minor modification pertains to the treatment of the fabric anisotropy. As discussed in several experimental studies and DEM analysis (Vaid et al. 1990; Arthur and Menzies 1972; Yoshimine et al. 1998; Yimsiri and Soga 2010), how particles assemble in a granular material has a dominating influence on the mechanical responses of the material. Owing to fabric anisotropy prevailing in sands, it is concluded that, for an undrained response, the direction of the major principal stress with respect to the fabric orientation $(\beta)$ and the value of the intermediate principal stress $\left(b=\frac{\sigma_{2}-\sigma_{3}}{\sigma_{1}-\sigma_{3}}\right)$, affect the mobilised stress paths significantly, particularly in the initial stages of the response. In order to consider the effect of varying dilation within the model skeleton, Dafalias et al. (2004) used a symmetric second order fabric tensor (F) and considered its interaction with the loading tensor (n) to arrive at a scalar measure of anisotropy $(A)$, as presented in Eq. (22). The value of $A$ (that would be constant for monotonic loading) was used for parallel translation of the CSL position (by changing $e_{0}$ in Eq. (1)). Other studies have tried scaling the CSL intercept using shape functions dependent on Lode angle (Shen et al. 2017). However, a series of later developments on this topic (Li and Dafalias 2012; Jefferies and Been 2016) have confirmed the uniqueness of the CSL. It is worth noting that the primary objective of these studies was to 
alter the onset of phase transformation (triggering contraction to dilation) by varying the absolute magnitude of $\psi$ in the model governing equations with respect to loading direction on inherent fabric.

The minor modifications to the initial version of the parent model (Dafalias and Manzari 2004) proposed here involves simple yet practical changes to circumvent the undesirable, variable CSL approach (see Eqs. (16), (23) to (26) in Table 1). In order to preserve the attributes of CSL constancy (Li and Dafalias 2012), the current model uses a constant CSL in e-p' space, contrary to the previous studies by Dafalias et al. (2004) and Papadimitriou et al. (2005), which adopt a loading and fabric tensor dependent CSL. The effect of varying mobilised stress and dilation with loading type is then introduced by adjusting the influence of $\psi$ (to $\psi_{f}$ in Eq. (26)) using scaling factors ( $f$ and $f_{h}$ in Eq. (24) and Eq. (25)) on the model's governing equations (Eq. (15), Eq. (16) and Eq. (20)). With the CSL kept unaltered, the approach of using a multiplicative factor on $\psi$ (i.e. $\psi_{f}$ ) allows the flexibility to accommodate the markedly varying responses for different loading directions with respect to fabric, with its effect progressively diminishing as the state gets closer to the CSL. In principle, this approach considers a variable rate of interaction of $\psi$ with the sand fabric for the different loading directions and serves to control the dilative and contractive tendencies of soil. In the version of the model adopted in this paper, anisotropy has been used in normalised form $\left(A_{\text {ratio }}=2 * \frac{A_{e}-A}{A_{e}-A_{c}}\right)$ (a modified form of Dafalias et al. 2004) so as to make it amenable to the form of the scaling function $\left(f\right.$ and $f_{h}$ ) adopted here (according to Eq. (23) to Eq. (26)). This practical approach of state parameter scaling would be suited for soil states not initially on the CSL (i.e. $\psi \neq 0$ ) and could serve as one the many ways to simulate the drastic differences in the initial stages of different monotonic stress paths having different fabric orientation angles $(\beta)$, but still allow the soil to ultimately attain the same $p^{\prime}$ at critical state conditions.

With respect to the relevance of the present work, it is worth noting that recent discrete element model studies have found that: i) the fabric tensor evolves towards a critical state value with plastic deformation (Li and Li 2009) and ii) large volume contractions occur on imposition of stress rotation at the critical state (Theocharis et al. 2017; Theocharis et al. 2019). Capturing the above effects compatibly under evolving fabric anisotropy can be achieved through the recently developed framework of ACST (Anisotropic Critical State Theory) (Li and Dafalias 2012), wherein an anisotropy dependent additive term was used to modify $\psi$ to control contractant versus or dilatant behaviour rather than the multiplicative factor adopted here (i.e. $\psi_{f}=f \psi$ ). Since the ultimate focus in this paper is to use a bounding-surface plasticity model for monotonic loading problems, the current simplified approach with a fixed fabric tensor and $\psi$ scaling is sufficient, without the need for the increased versatility of the 
later sophistications of the SANISAND family with evolving fabric that are targeted for strain accumulation with stress rotation of principal stress axes at fixed principal stress values (Petalas et al. 2019) or memory surface additions for cyclic ratcheting (Corti et al. 2016; Liu et al. 2018).

\section{Guidance for model parameter selection and their calibration}

This section provides guidance for selecting and calibrating the model parameters. The model (with the proposed modifications) has 18 parameters. The effect of each parameter on the model response, along with its calibration procedure, is outlined in Table 1 . Of the 18 parameters, the parameters $e_{0}, \lambda, \xi$, $M$ and $\mathrm{c}_{l}$, are mainly used to describe the position of the CSL. Three parameters, $G_{0}, v$ and $m$, mainly influence the elastic behaviour, whereas a group of six, $c_{2}, n^{b}, n^{d}, h_{i n}, c_{h}$, and $A_{0}$, influence the plastic behaviour and dilatancy. The fabric parameter $(a)$ and scaling parameters $\left(b_{1}, b_{2}\right.$ and $\left.k\right)$ play a key role in modifying dilatancy and $K_{p}$ in non triaxial compression (TXC) conditions.

The parameters are best calibrated in a hierarchical fashion. This may begin with accurate calibration of the five CSL parameters $\left(\mathrm{e}_{0}, \lambda, \xi, M\right.$ and $\left.\mathrm{c}_{1}\right)$, followed by the selection of three relatively trivial parameters $\left(m, c_{2}\right.$ and $v$ ). Of the remaining model parameters, $n^{b}, n^{d}, G_{0}, h_{i n}, c_{h}$, and $A_{0}$ (that mainly influence plastic behaviour) are calibrated using TXC test data using optimisation. A rational range for these parameters needs to be established before the optimisation technique is employed, as explained in subsequent sections. The fabric parameter $(a)$ and scaling factors $\left(f\right.$ and $\left.f_{h}\right)$ are subsequently determined on the basis of fits to undrained triaxial extension (TXE) or other anisotropic test data.

Laboratory data for two sands, UWA silica sand and Erksak sand, are used to demonstrate the process for selecting the parameters, and subsequently to demonstrate the merit of the model. The UWA silica sand test data are reported here (for the first time), whereas the Erksak sand test data were originally reported in Been et al. (1991). Geotechnical properties of the two sands are listed in Table 2.

\section{Calibration of the critical state parameters $\left(e_{0}, \lambda, \xi, M\right.$ and $\left.c_{1}\right)$}

Proper calibration of the critical state line (CSL) is of utmost importance since much of the model behaviour is functionally dependent on the state parameter $(\psi)$. The CSL can be determined using drained or undrained TXC test data on a set of loose or medium dense sands at different confining pressures sheared to deviatoric strain levels sufficient to achieve state constancy. If the model is being calibrated for use in a boundary 
value problem, it is important to calibrate the CSL accurately for the stress range expected in the boundary value problem.

The CSL equation used in the model is of the form $e_{c}=\mathrm{e}_{0}-\lambda\left(\frac{p^{\prime}}{p_{a t}}\right)^{\xi}$, where $e_{c}$ is the void ratio at critical state, $e_{0}, \lambda$ and $\xi$ are fitting parameters, and $p_{a t}$ is atmospheric pressure. As noted by Li and Wang (1998), $\xi$ has minor influence on the correlation and a value of 0.7 for sands worked well in most cases. Keeping $\xi$ fixed, the remaining two parameters, $e_{0}$ and $\lambda$, are established using least squares regression on data from each of the TXC tests sheared to the critical state. $e_{0}$ will always turn out to be close to $e_{\max }$ of the sand that is being calibrated (Klotz and Coop 2002).

The CSL points for UWA silica sand were chosen from eight TXC tests with sample relative densities $\left(R_{D}\right)$ ranging between $20 \%$ and $54 \%$ and $p^{\prime}$ at CSL ranging between $160 \mathrm{kPa}$ and $1350 \mathrm{kPa}$. The calibrated CSLs for UWA silica sand and Erksak sand are shown together with the test data in Fig. 2. The values of the critical stress ratio $(M)$ in TXC conditions can be computed from the average $q / p^{\prime}$ ratios at critical state and were found to agree well between samples tested at different void ratios and confining pressures. The parameter $c_{l}$ is the ratio of the critical stress ratio in TXE to the critical stress ratio in TXC and is generally found to lie between 0.63 and 0.83 . In the absence of reliable TXE data, $c_{l}$ may be set as $\frac{3-\sin \varphi_{c v}}{3+\sin \varphi_{c v}}$ (Sheng et al. 2000), where $\varphi_{c v}$ is the critical state friction angle in TXC conditions.

\section{Deciding on the value of the yield surface size (m)}

In order to successfully simulate plastic deformations during load reversals or unloading, the apex angle of the yield surface wedge has to be kept very small (Taiebat and Dafalias 2008). Based on previous reported calibrations, it was noted by that a value of the yield surface opening $(m)$ in the order of $M / 100$ works well for most sands. On the basis of this recommendation, $m$ has been chosen as 0.05 .

\section{Deciding on the initial size of bounding and dilatancy surfaces $\left(n^{b}, n^{d}\right)$}

The distances to the bounding and dilatancy surfaces from the current stress point (Fig.1) are influenced by $n^{b}$ and $n^{d}$. After calibration of the CSL, $M^{b}$ values can be plotted from the peak $q / p^{\prime}$ points of each of the TXC tests along with their corresponding $\psi$ values. A reasonable approximation for the parameter $n^{b}$ can then be obtained by fitting Eq. (11) to the plotted $M^{b}$ values. Similarly, an approximation of the parameter $n_{d}$ can be obtained using Eq. (12) fitting the $q-p^{\prime}$ and $\psi$ points at the phase transformation stage. However, owing to wide 
dispersion in the measured values across different data sets, as evident in Fig. 3 and Fig. 4, the values of $n^{b}$ and $n^{d}$ obtained in this fashion fail to reproduce a proper stress-strain fit for all the data sets. It is important to use these values as an initial guess and refine them through optimisation (later described) for an overall good fit.

\section{Calibration of the parameter $c_{2}$ controlling the gradient of the plastic potential surface}

For any deviatoric function of the form as suggested by Van Eekelen (1980), Potts and Gens (1984) established an expression for the Lode angle at critical state under undrained plane strain conditions. Noting that $\theta$ lies between $-30^{\circ}$ and $30^{\circ}$ for this expression (because of the inherent form used in Potts and Gens (1984) and based on the current deviatoric function $g_{2}\left(\theta, c_{2}\right)$ used here for plastic potential surface, the expression reduces to the form

$$
\tan \theta=\frac{-\frac{1-c_{2}}{1+c_{2}} \cos 3 \theta}{1+\frac{1-c_{2}}{1+c_{2}} \sin 3 \theta}, \theta=\tan ^{-1} \frac{2 b-1}{\sqrt{3}}
$$

A similar method was also used in the model calibration by Loukidis and Salgado (2009). Experiments on sands have shown that the value of $b$ lies close to 0.25 at critical state under plane strain conditions (Yoshimine et al. 1998). Solving the equation numerically would result in a value of $\theta$ close to $-16^{\circ}$, giving $c_{2}$ close to 0.8 .

\section{Deciding on the initial range for elastic parameters $\left(G_{0}, v\right)$}

The elastic shear modulus $(G)$ determined through Eq. (3) predominantly governs the initial (drained) stress-strain response and is influenced by the elastic constants $v$ and $G_{0}$. Rotisciani and Miliziano (2014) concluded that $v$ does not significantly affect the response in a drained TXC test. Based on the general guidelines provided by Benz (2007), any value of $v$ between 0.1 and 0.3 works reasonably well for sands, under drained conditions.

As the current model formulation does not consider the actual degradation of elastic shear modulus with strain level (Salgado et al. 2000), $G$ obtained using Eq. (3) serves mainly as an operative elastic shear modulus in the model and is expected to be significantly lower than the true maximum shear modulus $\left(G_{\max }\right)$. $G_{0}$ serves as a fitting constant for $G$, as well as influences $K_{p}$ (Eqs. (13) to (16)). Based on the initially assumed value of $v$, it is possible to match the drained $q-\varepsilon_{a}$ response reasonably at low $\varepsilon_{a}$ levels, e.g. $\varepsilon_{a} \lesssim 2.0 \%$ (a range which would encompass both plastic and elastic strains), by varying $G_{0}$ only. The initial response under drained conditions is relatively independent of the remaining plastic parameters, as evident in Fig. 5. $G_{0}$ fitted through this fashion provides a reasonable initial value for the optimisation process. 


\section{Deciding on a sensible range for the plastic parameters and dilatancy constants $\left(h_{i n}, c_{h}\right.$ and $\left.A_{0}\right)$}

The plastic parameters $h_{i n}$ and $c_{h}$ influence the plastic modulus in the model and have a pronounced influence on the point of phase transformation for an undrained triaxial response. Based on previously reported calibration data (Dafalias and Manzari 2004), the parameter $c_{h}$ seems to lie between 0.95 and 1.1 in most cases. For an initial guess before optimisation, $c_{h}$ may be assumed as 1.0 .

The initial value of $A_{0}$ can be guessed from the volumetric strain $\left(\varepsilon_{v}\right)$ and deviatoric strain $\left(\varepsilon_{q}\right)$ data of drained TXC along with the initial approximate position of $M^{d}$ by use of the following equation

$$
A_{o}=\frac{d \varepsilon_{v} / d \varepsilon_{q}}{\left(M^{d}-\eta\right)_{2}}=\frac{\frac{\left(\varepsilon_{v}\right)_{2}-\left(\varepsilon_{v}\right)_{1}}{\left(\varepsilon_{q}\right)_{2}-\left(\varepsilon_{q}\right)_{1}}}{\left(M^{d}-\eta\right)_{2}}
$$

where the respective points of 1 and 2 can be the phase transformation point and peak stress point, as shown in Fig. 7. Use of Eq. (28) gives initial values of $A_{0}$ of $0.585(=-0.177 /-0.303)$ for Erksak sand $\left(e_{0}=0.68\right.$ and $p_{0}{ }^{\prime}=$ $140 \mathrm{kPa})$ and $0.918(=-0.364 /-0.396)$ for UWA silica sand $\left(e_{0}=0.6845\right.$ and $\left.p_{0}{ }^{\prime}=80 \mathrm{kPa}\right)$.

Using the approximate value of $G_{0}$ obtained in the previous section, an approximate value of $h_{\text {in }}$ can be established by matching the phase transformation point observed in an undrained TXC test, as shown in Fig. 6. As $h_{i n}$ also influences the gradient of the $q-\varepsilon_{a}$ plot close to peak stresses for a drained response (Fig. 5), it needs to be refined through optimisation.

\section{Use of optimisation technique to calibrate parameters for TXC datasets}

In order to implement the model for a boundary value problem, a single set of model parameters is required to define a given soil. Experience of working with the model indicated that the CSL parameters were unique and relatively easy to obtain. However, a single model set of (mostly) plastic parameters, effectively matching every measured data set, is difficult to determine, which may be due to variations across samples, or slight inconsistencies in test quality. A single parameter may even influence multiple key attributes in an element test simulation, and hence cannot be derived by strictly bracketing it to capture only one distinct feature. In such cases, the optimisation routine helps to produce the most rational set of parameters by minimisation of a framed objective function, thus producing a better overall fit to the entire TXC data set. The fit of the simulated plots in both stress and deformation spaces, normalised with respect to the absolute maximum values on the axis, has been considered for optimisation across all the measured data sets. This removes the subjectivity and improves the confidence of 
parameter determination rather than choosing them from a single set. For drained TXC tests, $q / p^{\prime}$ vs $\varepsilon_{a}$ and $\varepsilon_{v}$ vs $\varepsilon_{a}$ space has been considered, whereas for undrained TXC tests, $q$ vs $\varepsilon_{a}$ and $q v s p^{\prime}$ space has been considered.

The objective function $(C)$ used is similar to that described in Mattsson et al. (2001) and used successfully by Doherty et al. (2012). It is formed by summing up the minimum distance $\left(d_{\min }^{i}\right)$ between each individual point on an experimental curve and the numerically generated curve, expressed as

$$
C=\frac{\sum_{i=1}^{n} w_{i} d_{\text {min }}^{i}}{\sum_{i=1}^{n} w_{i}}
$$

where $n$ is the total number of experimental data points and $w_{i}$ is the weight assigned to each data point. For each experimental data point (denoted as $i$ in Fig. 8), the current optimisation technique involves a search to find the set of closest triplets $\left(x_{j-1}, x_{j}, x_{j+1}\right)$ on the simulated curve. This is equivalent to locating the set of $x_{j-1}, x_{j}$ and $x_{j+1}$ such that, for the calculated linear distances shown in Fig. $8, d_{j}^{i}<d_{j-1}^{i}$ and $d_{j}^{i}<d_{j+1}^{i}$ follows. Since the process was carried out with a simulated response of higher resolution than the measured response, $d_{\min }^{i}$ was subsequently approximated as the mean of linear distances from the closest triplet $\left(d_{\min }^{i}=\left(d_{j-1}^{i}+d_{j}^{i}+d_{j+1}^{i}\right) / 3\right)$. The process is then repeated for the next experimental data point, with the subsequent search for the closest triplet commencing at the $x_{j+2}^{\text {th }}$ value on the simulated curve. If certain sections of the measured response are to be prioritised for a given fit, higher weights (i.e. values of $w_{\mathrm{i}}$ ) may be used for those sections. Care is needed when computing distances at the stationary points (e.g. at the start of softening, dilation etc.).

At the start of optimisation, the rational range for $G_{0}, n^{b}, n^{d}, h_{i n}, c_{h}$ and $A_{0}$ may be taken as $\pm 50 \%$ of the initial values decided above, so as to discover the most likely range for the fitting set of parameters. For successful use of the technique, the experimental values used for framing the objective function should be considered prior to the point where shear bands start to form. A nested optimisation technique is then used, with the set of fitting parameters varied incrementally within the specified rational range. The parameter set that provides the minimum value of $C$ within the previously decided range is finally chosen (as illustrated by the dashed lines in Fig 3 and 4).

\section{Use of state parameter scaling function to compute non -TXC parameters}

After optimising the TXC parameters, the focus shifts to calibrating the non-TXC parameters. Within the constitutive model framework, the increasingly softer response as the loading conditions change from triaxial compression $\left(A_{\text {ratio }}=2\right.$, see Eq. $\left.(23)\right)$ to triaxial extension $\left(A_{\text {ratio }}=0\right)$ is achieved by modifying the influence of $\psi$ on $D$ using scaling factors. The newly introduced scaling factor $(f)$ (cast in terms of $A_{\text {ratio }}$ in Eq. (23)) is 
calculated through Eq. (24) to cause a favourable change of $D$ in Eq. (20) as $A_{\text {ratio }}$ changes from 2 to 0 . Although it also serves to reduce $K_{p}$ due to a reduction in $M_{\theta}^{b}$ (in Eq. 12), a stronger dependence was necessary to scale down $K_{p}$ using $f_{h}$ (Eq. (25) and Eq. (13-16))to simulate the effect of higher softening and match element test data better. Such an approach is consistent with the concept of different phase transformation lines for TXC and TXE observed in experiments (Riemer and Seed 1997; Jefferies and Been 2016). The inherent nature of Eq. (24) means that the values of $f$ and $f_{h}$ are triggered only during non-TXC conditions and permits the fabric and scaling factor constants to be fitted based on TXE tests.

$A_{\text {ratio }}$ in the scaling functions (Eq. (23)) is influenced by the fabric parameter $a$ in Eq. (21), where $a$ is an indication of the preferential particle orientation of the sand. A value of $a$ equal to 0 would simulate a situation where particles lie entirely on the horizontal bedding plane, whereas $a$ equal to 0.33 would mean an isotropic fabric (Dafalias et al. 2004). Papadimitriou et al. (2005) showed that the value of $a$ used to define the fabric tensor (F) is typically expected to lie between 0.27 and 0.33 for different sample preparation methods. Based on evidence that samples prepared using wet pluviation leads to preferential horizontal deposition similar to natural soil deposits (Vaid et al. 1999), the parameter $a$ for UWA silica sand in the model being considered here was chosen as 0.27 . However, in the model being considered here, the use of anisotropy in normalised form as obtained through Eq. (23) reduces the direct impact of $a$ significantly. In Eq. (24) and (25), $b_{l}$ and $k$ are determined initially by fitting stress-strain responses ( $q$ vs $\varepsilon_{a}, q v s p^{\prime}$ ) to TXE test data for the case of $A_{\text {ratio }}=0$. Parameter $b_{2}$ is introduced to retain the flexibility of fitting $f$ to match an inherently anisotropic test, where $b$ takes values between 0 and 1 , and $\beta$ takes values between $0^{\circ}$ and $90^{\circ}$, resulting in different (but constant) values of $A_{\text {ratio }}$ lying between 0 and 2. The value of $b_{3}$ is decided according to Eq. (24), so as to make $f$ equal to 1.0 when $A_{\text {ratio }}$ is equal to 2, thus maintaining the calibration already achieved for TXC conditions.

\section{Model prediction for drained and undrained triaxial tests}

The efficiency of the proposed calibration procedure is demonstrated by way of numerical simulations of isotropically consolidated $\left(K_{0}=\sigma_{h}{ }^{\prime} / \sigma_{v}{ }^{\prime}=1.0\right)$ drained and undrained triaxial compression tests for the Erksak and UWA sands considered in the parameter determination section using the optimised parameters listed in Table 3. Results of these simulations are compared with the measurements in Fig. 9 and Fig. 10 for triaxial compression tests. 
Fig. 9 shows that the bounding surface model can adequately capture peak stress and post peak softening responses in Erksak sand for drained and undrained triaxial compression tests for a wide range of confining pressures and densities. However, a slight under-prediction of volumetric strain can be observed for the test with $e_{0}=0.691 \sigma_{v}{ }^{\prime}=130 \mathrm{kPa}$ and $e_{0}=0.677, \sigma_{v}{ }^{\prime}=60 \mathrm{kPa}$ (Fig. 9(b) and Fig. 9(c)). This may be due to slight uncertainty on the CSL parameters, evident from the scatter at low $p^{\prime}$ levels in Fig. 2(a). A higher CSL intercept $\left(e_{0}\right)$, causing a more dilatant response, would improve the simulations at lower stress levels, noting that these minor adjustments may also result in final critical state void ratios being slightly higher than $e_{\max }$.

Fig. 10, which compares simulations and measurements for the UWA tests, shows an apparent deviation of $q$ at critical state for a few cases by $\pm 11 \%$, as $M$ from experimental data varied between 1.15 and 1.3 . This was probably due to the formation of shear bands in the samples at higher strain levels causing possible inhomogeneity in the overall response. Shear band formation is to be expected as the triaxial samples were prepared using the air pluviation technique to maintain consistency with the centrifuge tests considered later in the paper (Section 5).

Nevertheless, the value of $M$ was chosen as 1.296 for UWA sand corresponding to a $\phi_{c v}$ of $32.2^{\circ}\left(M=\frac{6 \sin \phi_{c v}}{3-\sin \phi_{c v}}\right)$, which is in the range reported in the literature for the same sand (Teh et al. 2010; Chow et al. 2019). As noted earlier in the paper, the optimisation technique was typically undertaken up to the point where shear bands started to form, typically observed at strain levels of $\varepsilon_{a} \sim 13 \%$ in UWA silica sand. Despite some level of apparent uncertainty in $M$, the simulations provide very good agreement with the measurements in predicting peak stress, post peak softening and volumetric dilation up to strain levels of $\varepsilon_{a}=12$ to $14 \%$. The inset in Fig. 10(c) shows that the optimisation technique is able to capture $p^{\prime}$ at the point of phase transformation within a margin of $7.4 \%$ and $2.2 \%$ respectively for the two different tests.

In order to demonstrate the usefulness of the optimisation technique, the optimised parameter set was used to simulate the responses of four separate (both isotropically and anisotropically consolidated) drained compression triaxial tests on UWA silica sand with a wider range of vertical confining stresses $\left(\sigma_{v}{ }^{\prime}=40\right.$ to 1000 $\mathrm{kPa}$ and $\left.K_{0}=0.5\right)$ as summarised in Table 4 . The optimised parameter set, being more robust and rationally attained, is seen (Fig. 11) to capture the responses to within 3\%. The volumetric responses were also reasonably accurate, with slight deviations noticed in Test 1 because of localisation. On the other hand, if the optimisation technique had not been used and the parameters were determined through individual best fitting approaches to these tests, the important parameters $\left(G_{0}, h_{i n}, A_{0}, n^{b}\right.$ and $\left.n^{d}\right)$ would have showed a greater degree of variability as demonstrated by the higher coefficient of variation (COV) in Table 4. 
To demonstrate the importance of the fabric anisotropy consideration, Fig. 12 compares simulations (with and without anisotropy consideration) for undrained TXE tests on UWA silica sand at two different confining pressures. Due to necking, the tests did not reach critical state conditions and the experimental responses are shown up to $\varepsilon_{\mathrm{a}} \approx 8 \%$. Since the TXE tests in UWA silica sand did not reach critical state conditions, the value of $c_{l}$ for the calibration was taken to be 0.7 based on $\phi_{\mathrm{cv}}$ of $32.2^{\circ}$ (Sheng et al. 2000). As tests at different fabric orientations are not available for UWA silica sand, $f=0.6$ (for $A_{\text {ratio }}=1$ ) has been chosen to maintain consistency with non-linear calibration trends reported in Loukidis and Salgado (2009), which would have given $b_{2}=-0.833$. Unlike the simulation without anisotropy consideration, the scaling functions $f$ and $f_{h}$ based on $A_{\text {ratio }}$ were able to simulate the initial contractant response and the varying dilation rate, leading to satisfactory predictions (to varying degree) for strain levels up to the point of phase transformation in the undrained TXE tests.

To further investigate the appropriateness of the proposed inherent anisotropic scheme, Fig 13(a) presents the simulated responses under the same loading of $p^{\prime}=150 \mathrm{kPa}$ for different fabric orientations. The simulations show increasingly contractive behaviour (particularly at low shear strain levels) as the fabric orientation $(\beta)$ increases from $0^{\circ}$ to $90^{\circ}$ and intermediate principal stress $(b)$ changes from 0 to 1 . A qualitative comparison with the simulations presented in Dafalias et al. (2004) suggests that the current model would produce a similar softening in the initial response, but attain the same $q$ value at large strains (Fig 13(b)), owing to constant CSL assumed in the model.

Fig. 14 compares the model performance in simulation of simple shear tests. Although the simulated response is slightly higher than the measured data, the beneficial effect of state parameter scaling is evident in the initial stages of the response. Further scaling by starting with an even lower minimum value of $f$ or $f_{h}$ would be needed to match the measurements at higher shear strains. This could have been achieved by assigning slightly higher values for $b_{l}$ and $k$ (thus lowering $f$ or $f_{h}$ at $A_{\text {ratio }}=0$ as per Eqns. (23-25)), but any changes in these parameters would have significantly under-predicted the TXE tests lying at the extreme end of the load spectrum.

\section{Implementation of the model in FE analyses of boundary value problems}

Having demonstrated the ability of the model to simulate responses measured in element tests adequately, this section examines the ability of the model to simulate the stress and deformation response in boundary value problems. Two different boundary value problems on the same UWA silica sand (as examined in the element tests) are selected here: (a) a buried horizontal circular plate anchor and (b) a circular surface footing, both loaded vertically. 
The anchor and footing tests are reported in detail in Hao et al. (2018) and White et al. (2008) respectively, with a brief summary provided here. Both sets of tests were conducted in a geotechnical centrifuge in dry UWA silica sand. The anchor tests were conducted at $20 \mathrm{~g}$ using 'wished in place' circular plates with a diameter of 20 $\mathrm{mm}$ and a thickness of $2 \mathrm{~mm}$ (representing an equivalent prototype anchor with a diameter, $D=0.4 \mathrm{~m}$ and thickness, $t=0.04 \mathrm{~m}$ ) in dry sand of $R_{D}$ of $85 \%$. The plate anchor tests were at embedment depths of between $3 D$ and $12 D$ and were loaded to a vertical displacement of (typically) $0.4 D$. The footing tests were conducted at $40 \mathrm{~g}$ using a footing with a diameter of $60 \mathrm{~mm}$ and thickness of $7 \mathrm{~mm}$ (such that the equivalent prototype dimensions were $D=2.4 \mathrm{~m}$ and $t=0.28 \mathrm{~m}$ ). The relative density of the sand in the footing tests was either $R_{D}=54 \%$ or $R_{D}$ $=78 \%$. The footing was located on the sand surface and loaded to a footing settlement of $0.35 D$.

\section{Details of the numerical model}

The corresponding numerical simulations were carried out as axisymmetric analyses in the commercial FE software package, Abaqus. Both the anchor and footing were modelled as rigid bodies with the same prototype dimensions as in the centrifuge experiments. The sand was modelled with the same model parameters adopted in the simulation of the element tests (provided in Table 3) and the unit weight $\left(\gamma^{\prime}\right)$ of the sand in the simulations was kept identical to that reported in the experiments. A schematic diagram of the FE model with a representative mesh is shown in Fig. 15. The analyses used a graded unstructured mesh composed of linear quadrilateral elements (CAX4) with a minimum element size of $0.02 D$ for the footing and anchor problem at the soil-structure interface. It is acknowledged that failure loads obtained for a boundary value problem using a strain softening model can depend on mesh discretisation. Although mesh regularisation techniques, e.g. nonlocal regularisation (Summersgill et al. 2017), have not been considered here (as it is beyond the scope of this study), a mesh sensitivity analysis showed that subsequent mesh refinements do not alter the peak failure loads because of the predominantly bearing dominated nature of the problem (Loukidis and Salgado 2011).

The vertical and lateral boundaries of the domain for the footing simulations were taken as $5 D$ and $6 D$ respectively from the centre of the footing. For the anchor problem, the bottom boundary was fixed at $3 D$ below the anchor whereas the lateral boundary was varied to maintain a distance sufficient to avoid boundary effects. Although the interface roughness of the centrifuge models was not specified, the interface between the footing base and the soil was modelled using surface to surface formulation following a penalty method with a friction coefficient of $0.7\left(\sim \tan 35^{\circ}\right)$. The choice of interface roughness has a very minor influence on the capacity of a buried horizontal plate subjected to vertical loading (Merifield and Sloan 2006), such that the interface between 
the anchor and the soil was taken to be fully rough. The coefficient of earth pressure $\left(K_{0}\right)$ for both cases was taken to be 0.5 .

\section{Constitutive model integration scheme methodology}

An explicit integration scheme based on the formulation described in Sloan et al. (2001) was implemented to obtain the updated stresses for a given increment of strain. At each Gauss point, the ratio of the pure plastic strain to the total strain was obtained by solving for yield surface intersection using Pegasus method (Sloan et al. 2001). The plastic stresses were then obtained according to the Modified Euler scheme for the computed plastic strains using a pseudo time $T(0<T<1)$ with increments of substep size $(d T)$. As suggested in Sloan et al. (2001), the relative errors were then checked against a substepping tolerance, followed by an iterative drift correction in each substep. The yield surface tolerance used for the boundary value problem was $0.001 \mathrm{kPa}$ and the substepping tolerance was $10^{-5}$. On a global scale, the elastic stiffness matrix at the point of updated stress was taken for equilibrium iterations. The maximum size of the global time increments was kept at $0.0001 D$ to allow convergence with five to six global iterations.

A few other modifications were necessary for the model implementation in the FE analyses. A threshold value of $p^{\prime}$ was set at $0.001 \mathrm{kPa}$. If after updating the stresses using the modified Euler method, $p^{\prime}$ fell below the threshold limit, the stress state was kept unchanged for that increment at that Gauss point. The minimum value of $d T$ allowed for the problem was $10^{-10}$. It was noticed that in areas of intense shear band development close to the footing or anchor, the value of $d T$ was very close to the minimum allowed value of $10^{-10}$, which would result in very high runtimes. For computational efficiency in such cases, if $d T$ was $10^{-7}$ or less, a substepping error tolerance of $10^{-3}$ was used instead of $10^{-5}$ for the current $d T$. Drifting of the yield surface was corrected according to the consistent drift correction method suggested in Sloan et al. (2001). During drift correction, if divergence was observed, the consistent drift correction method was ignored and the stresses were instead made to converge using the method of normal drift correction (Sloan et al. 2001). Explicit FE approaches based solely on normal drift correction have also been used by (Tian and Cassidy 2010). This (pragmatic) approach allowed the FE software to override discontinuities encountered at a few discrete Gauss points without resulting in prematurely aborted simulations.

The finite element formulations also produced a negative plastic multiplier in some cases at a few Gauss points, due to overshooting (Dafalias and Taiebat 2016). In such scenarios, $d T$ was reduced by a factor of four before undertaking the next trial. If the negative value of plastic multiplier persisted even after $d T$ was reduced to 
$10^{-7}$ or less, the elastic matrix at the current stress state was used for the current update (Sloan et al. 2001) using the Modified Euler scheme followed by drift correction. This approach was considered reasonable as this only arose for very few Gauss points.

\section{Numerical results from the boundary value problems}

Fig. 16 compares the results from the footing numerical simulations with the experimental measurements. The results are presented as a mobilised bearing factor $\left(N_{\gamma}=\frac{2 q_{u}}{\gamma^{\prime} D}\right)$ against normalised settlement $(\delta / D)$, where $q_{u}$ is the bearing pressure. Fig. 16 shows that strain softening was only observed in the experiments at $R_{D}=78 \%$, whereas the simulations indicate varying degrees of strain softening at both relative densities. The simulation for $R_{D}=54 \%$ gave a peak capacity at $\delta / D=5 \%$, before reducing by $16.8 \%$ at $\delta / D=15 \%$, whereas the simulation for $R_{D}=78 \%$ gave a peak capacity at $\delta / D=4.1 \%$, with a reduction of $27.6 \%$ at $\delta / D=15 \%$. The higher percentages of post-peak reductions with increasing $R_{D}$ agree well with the typical strip footing simulations presented in Chaloulos et al. (2019). It is important to note that footing capacity is over predicted by a factor of 2.0 to 2.5 when the simulations do not use the fabric anisotropic factors. Similar overpredictions were also observed in the strip footing simulations reported by Chaloulos et al. (2019), and demonstrate the importance and necessity of macroscopic incorporation of fabric effects in sand when analysing a boundary value problem, which ultimately leads to proper consideration of all shearing modes (i.e. TXC and non-TXC) during element level model calibration.

Although the initial mobilisation response in the footing problem is not captured well by the simulations, the peak simulated $N_{\gamma}$ agrees well with the peak measured values, being within $1.6 \%$ and $4.1 \%$ for the $R_{D}=54 \%$ and $78 \%$ cases respectively. Also shown on Fig. 16 is the back-calculated friction angle $(\varphi)$, corresponding to the peak experimental capacity for the two tests, obtained using the slip-line method (Martin 2005). This backcalculation gave $\varphi=36^{\circ}$ and $38^{\circ}$ for the tests at $R_{D}=54 \%$ and $78 \%$ respectively. It is instructive to examine what peak friction angle a practitioner might have chosen for this problem. For instance, adopting (Bolton 1986) correlations would result in $\varphi=37.8^{\circ}$ for $R_{D}=54 \%$ and $40.7^{\circ}$ for $R_{D}=78 \%$ (assuming $p^{\prime}=0.1 q_{u}$ ), which is $\sim 2^{\circ}$ and $\sim 3^{\circ}$ higher than the respective back-calculated values. Applying the same slip-line method using these friction angles would result in an overestimation in $N_{\gamma}$ of $38.7 \%$ for the $R_{D}=54 \%$ test and $74.1 \%$ for the $R_{D}=78 \%$ test. This simple exercise emphasizes the potential level of inaccuracy associated with simple models that rely on a single lumped input parameter and ignore the nature of progressive failure that may dominate the problem. 
As noted previously, the surface footing simulations gave an initial response that was much stiffer than the measured response, consequently resulting in poorer agreement between the measured and simulated $N_{\gamma}$ at a given normalised displacement. For instance at $\delta / D=2.0 \%$, which is a typical serviceability limit for footings, the simulated $N_{\gamma}$ values are 2.45 and 1.6 times higher than the measured responses for the $R_{D}=54 \%$ and $R_{D}=78 \%$ cases respectively. This is likely to be because the current model uses a lower operative elastic shear modulus than the true $G_{\max }$ of the soil and does not consider the actual degradation of elastic shear modulus with strain level (Salgado et al. 2000). To investigate this further, two additional footing analyses were undertaken for the case with $R_{D}=78 \%$ in which $G_{0}$ and $h_{i n}$ were modified in an attempt to alter the initial stiffness. In the first analysis $G_{0}$ and $h_{i n}$ were varied proportionately in the model by $33 \%$ (case A in Fig. 17), so as to maintain the same $K_{p}$, whereas in the second analysis only $G_{0}$ was reduced by $33 \%$ (case B in Fig. 17). Fig. 17 shows that proportionally adjusting $G_{0}$ and $h_{\text {in }}$ to maintain the same $K_{p}$ (case A) led to a slight improvement in the initial stiffness, whilst maintaining approximately the same peak $N_{\gamma}$. Reducing only $G_{0}$ (case B) led to an initial stiffness that best matched the measurements. However, this better agreement in the initial footing response was at the cost of under predicting the peak $N_{\gamma}$ by $14.3 \%$, since $G_{0}$ also influences the plastic parameters in the model through Eq. $13-15$. Although proportional adjustment of $G_{0}$ and $h_{i n}$ may appear justifiable, the effect for other boundary value problems involving different stress paths would need to be considered, noting that these adjustments also worsen the simulation of element tests.

Fig. 18 compares the back-analysed operative shear modulus for the measured and simulated footing responses using mobilised bearing pressure $\left(q_{m o b}\right)$ at $R_{D}=78 \%$ up to initial $\delta / D=2 \%$ based on the elastic solutions given by Mayne and Poulos (1999). The measured responses correspond to an operative $G=11.2 \mathrm{MPa}$, whereas the simulated responses correspond to $G=20.5 \mathrm{MPa}$. The expected operative shear modulus can be estimated from the small strain shear modulus $\left(G_{\max }\right)$ using the shear modulus degradation expression in Oztoprak and Bolton (2013):

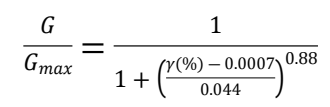

where the shear strain, $\gamma$ is expected to be in the range $0.05-0.2 \%$ for a footing (Likitlersuang et al. 2013). Using $G_{\max }=71 \mathrm{MPa}$ for the UWA silica sand (obtained by assuming $p^{\prime} \sim 0.1 q_{\operatorname{mob}}$ at $\delta / D=1.5 \%$ ), the operative shear modulus for a footing at $\gamma=0.13 \%$ would be $G=19.8 \mathrm{MPa}$, close to that for the simulations. Hence, the (prefailure) simulated response is consistent with elastic solutions in the initial stage, such that the stiffness response is not a characteristic deficiency of the constitutive model on the whole. 
Fig. 19 compares the numerical simulations with the measured responses for a buried circular plate anchor at an embedment ratio $(H / D)$ of 3 for $R_{D}=85 \%$. Capacity is presented in dimensionless form using an anchor factor $\left(N_{\gamma}=\frac{q_{u p}}{H \gamma^{\prime}}\right.$, where $q_{u p}$ is the uplift capacity). The simulations, although slightly stiffer than the measured responses by a factor of 1.14 up to $\delta / D=2 \%$, capture the peak capacity reasonably well, and are within $5 \%$ of the measured responses. By way of comparison, Fig. 19 also shows plate anchor capacity calculated using an axisymmetric version of the plane strain limit equilibrium method proposed in White et al. (2008) for buried strip anchors and pipelines. Details of the axisymmetric limit equilibrium method are given in Hao et al. (2018) and adopt peak friction and dilation angles based on Bolton's $(1986,1987)$ stress-dilatancy correlations. The limit equilibrium calculations significantly under predict the measured responses in this case because of the oversimplistic assumptions of mobilised friction angles in the calculation of shear stresses along the slip surfaces ${ }^{1}$. As also shown by Fig. 19, improvements to the calculated anchor capacity can be obtained by making more realistic assumptions on the shear stresses developed along the slip surfaces, as allowed for in the Giampa et al. (2017) limit equilibrium approach to the same problem. The level of agreement is now within $12.5 \%$ and is on a par with the finite element simulations.

\section{Conclusions}

The suitability of a bounding surface plasticity model with pertinent modifications has been investigated here using element test responses from UWA silica sand and Erksak sand, followed by numerical analysis of boundary value problems. The current version uses a constant CSL and captures strength anisotropy by varying influence of the state parameter on dilatancy and hardening modulus. The varying influence has been introduced through use of scaling factors that are based on a normalised measure of relative interaction between fabric and the loading direction. A systematic calibration method using an optimisation technique has been introduced to produce the most rational set of model parameters. The results show that the model is able to satisfactory capture the effect of soil density and stress level dependency through a unique set of 18 parameters. The model also captures triaxial extension (TXE) responses reasonably well up to axial strain levels of around $8 \%$.

Application of the model using calibrated parameters in finite element simulations of a simple surface footing and buried circular anchor in UWA silica sand showed that the predicted normalised capacities are within a maximum margin of $4.1 \%$ and $5 \%$ in the two cases respectively. On the contrary, footing capacity would have

${ }^{1}$ The reported differences are higher than reported in Hao et al. (2018) as the measured uplift factors were corrected for the reduced overburden stress due to the rigid loading shaft 
been overpredicted by 2.0 to 2.5 times if anisotropic factors were not considered. Capturing the appropriate stiffness response in the initial stages was challenging for the surface footing problem with corrective attempts having a detrimental effect on mobilised peak capacity. However, despite the mismatch in the pre-peak behaviour for footings, the model is superior in calculating absolute values of capacity relative to simple models. This also demonstrates that a shift in engineering practice and research endeavour is needed towards proper analysis and accurate design of geotechnical structures using advanced constitutive models rather than relying on empirical methods, which do not capture all aspects of interaction.

However, the set of 18 model parameters requires high quality laboratory data for calibration, making it of limited appeal from a practical point of view. Extensive research focussing on creating a broader database for sand calibration followed by subsequent development of direct correlations linking the routinely used sand properties with some of the model parameters would help a long way in elucidating the model. The present model was adapted with a focus on capturing monotonic behaviour of sands. Additional modifications are likely to be required for modelling cyclic loading.

\section{Acknowledgements}

This work was supported by the Lloyd's Register Foundation. The Lloyd's Register Foundation invests in science, engineering and technology for public benefit, worldwide.

\section{References}

Arthur, J.R.F., and Menzies, B.K. 1972. Inherent Anisotropy in a sand. Géotechnique, 22(1), pp.115-128.

Been, K., Jefferies, M.G., and Hachey, J. 1991. The critical state of sands. Géotechnique, 41(3), pp.365-381.

Bolton, M.D. 1986. The strength and dilatancy of sands. Géotechnique, 36(1), pp.65-78.

Chaloulos, Y.K., Papadimitriou, A.G., and Dafalias, Y.F. 2019. Fabric Effects on Strip Footing Loading of Anisotropic Sand. Journal of Geotechnical and Geoenvironmental Engineering, 145(10), pp.1-15.

Chow, S.H., Roy, A., Herduin, M., Heins, E., King, L., Bienen, B., O' Loughlin, C.D., Gaudin, C., and Cassidy, M.J. 2019. Characterisation of UWA superfine silica sand, Ocean Graduate school Technical Report, The University of Western Australia, GEO 18844

Corti, R., Diambra, A.,Wood, D.M., Escribano, D.E., and Nash, D.V.T 2016. Memory surface hardening model for granular soils under repeated loading conditions. Journal of Engineering Mechanics, 142(12), pp.1-14. 
Dafalias, Y.F., and Manzari, M.T. 2004. Simple Plasticity Sand Model Accounting for Fabric Change Effects. Journal of Engineering Mechanics, 130(6), pp.622-634.

Dafalias, Y.F., Papadimitriou, A.G., and Li, X.S. 2004. Sand plasticity model accounting for inherent fabric anisotropy. Journal of Engineering Mechanics, 130(11), pp.1319-1333.

Dafalias, Y.F., and Popov, E.P. 1975. A model of nonlinearly hardening materials for complex loading. Acta Mechanica, 21(3), pp.173-192.

Dafalias, Y.F., and Taiebat, M. 2016. SANISAND-Z: zero elastic range sand plasticity model. Géotechnique, 66(12), pp.999-1013.

Doherty, J., Alguire, H., and Muir Wood, D. 2012. Evaluating modified Cam clay parameters from undrained triaxial compression data using targeted optimization. Can. Geotech. J., 49(11), pp.1285-1292.

Drucker, D.C., and Prager, W. 1952. Soil mechanics and plastic analysis or limit design. Quarterly of Applied Mathematics, 10(2), pp.157-165.

Duncan, J.M., and Chang, C.Y. 1970. Nonlinear Analysis of Stress and Strain in Soil. ASCE Journal of the Soil Mechanics and Foundations Divi- sion, 96(5), pp.1629-1653.

Van Eekelen, H.A.M. 1980. Isotropic yield surfaces in three dimensions for use in soil mechanics. International Journal for Numerical and Analytical Methods in Geomechanics, 4(1), pp.89-101.

Gajo, A., and Wood, D.M. 1999. A kinematic hardening constitutive model for sands: the multiaxial formulation. International Journal for Numerical and Analytical Methods in Geomechanics, 23, pp.925-965.

Giampa, J., Bradshaw, A., and Schneider, J. 2017. Influence of Dilation Angle on Drained Shallow Circular Anchor Uplift Capacity. International Journal of Geomechanics, 17(2), p.4016056.

Grammatikopoulou, A., Zdravkovic, L., and Potts, D.M. 2007. The effect of the yield and plastic potential deviatoric surfaces on the failure height of an embankment. Géotechnique, 57(10), pp.795-806.

Gudehus, G. 1996. A comprehensive constitutive equation for granular materials. Soils and Foundations, 36(1), pp.1-12.

Hao, D., Wang, D., O’Loughlin, C.D., and Gaudin, C. 2018. Tensile monotonic capacity of helical anchors in sand: interaction between helices. Canadian Geotechnical Journal, 3483, pp.1-33. 
Klotz, E.U., and Coop, M.R. 2002. On the Identification of Critical State Lines for Sands. Geotechnical Testing Journal, 25(3), pp.289-302.

Li, X., and Li, X.S. 2009. Micro-macro quantification of the internal structure of granular materials. Journal of Engineering Mechanics, 135(7), pp.641-656.

Li, X.S., and Dafalias, Y.F. 2012. Anisotropic Critical State Theory: Role of Fabric. Journal of Engineering Mechanics, 138(3), pp.263-275.

Li, X.S., and Dafalias, Y.F. 2002. Constitutive modeling of inherently anisotropic sand behavior. Journal of Geotechnical and Geoenvironmental Engineering, 128(10), pp.868-880.

Li, X.S., and Dafalias, Y.F. 2000. Dilatancy for cohesionless soils. Géotechnique, 50(4), pp.449-460.

Li, X.S., and Wang, Y. 1998. Linear Representation of Steady-State Line for Sand. Journal of Geotechnical and Geoenvironmental Engineering, 124(12), pp.1215-1217.

Likitlersuang, S., Teachavorasinskun, S., Surarak, C., Oh, E., and Balasubramaniam, A. 2013. Small strain stiffness and stiffness degradation curve of Bangkok Clays. Soils and Foundations, 53(4), pp.498-509.

Liu, H.Y., Abell, J.A., Diambra, A., and Pisano, F. 2018. Modelling the cyclic ratcheting of sands through memory-enhanced bounding surface plasticity. Géotechnique, (9), pp.1-18.

Loukidis, D., Chakraborty, T., and Salgado, R. 2008. Bearing capacity of strip footings on purely frictional soil under eccentric and inclined loads. Canadian Geotechnical Journal, 45(6), pp.768-787.

Loukidis, D., and Salgado, R. 2011. Effect of relative density and stress level on the bearing capacity of footings on sand. Géotechnique, 61(2), pp.107-119.

Loukidis, D., and Salgado, R. 2009. Modeling sand response using two-surface plasticity. Computers and Geotechnics, 36(1-2), pp.166-186.

Manzari, M.T., and Dafalias, Y.F. 1997. A critical state two-surface plasticity model for sands. Géotechnique, 47(2), pp.255-272.

Martin, C.M. 2005. Exact bearing capacity calculations using the method of characteristics. Proceedings of the 11th International Conference of IACMAG, 4(441-450).

Mattsson, H., Klisinski, M., and Axelsson, K. 2001. Optimization routine for identification of model parameters 
in soil plasticity. International Journal for Numerical and Analytical Methods in Geomechanics, 25(5), pp. $435-472$.

Mayne, P.W., and Poulos, H.G. 1999. Approximate Displacement Influence Factors for Elastic Shallow Foundations. Journal of Geo- technical and Geoenvironmental Engineering, 125(6), pp.453-460.

Merifield, R.S., and Sloan, S.W. 2006. The ultimate pullout capacity of anchors in frictional soils. Canadian Geotechnical Journal, 43(8), pp.852-868.

Oztoprak, S. , and Bolton, M.D. 2013. Stiffness of sands through a laboratory test database. Géotechnique, 63(1), pp.54-70.

Papadimitriou, A.G., Dafalias, Y.F., and Yoshimine, M. 2005. Plasticity modelling of the effect of sample preparation method on sand response. Soils and Foundations, 45(2), pp.109-123.

Petalas, A.L., Dafalias, Y.F., and Papadimitriou, A.G. 2019. SANISAND-FN: An evolving fabric-based sand model accounting for stress principal axes rotation. International Journal for Numerical and Analytical Methods in Geomechanics, 43(1), pp.97-123.

Potts, D.M., and Gens, A. 1984. the Effect of the Plastic Potential in Boundary Value Problems Involving Plane Strain Deformation. International Journal for Numerical and Analytical Methods in Geomechanics, 8(259286).

Riemer, M.F., and Seed, R.B. 1997. Factors affecting apparent position of steady-state line. Journal of Geotechnical and Geoenvironmental Engineering, 123(March), pp.281-288.

Roscoe, K.H., Schofield, A. N., and Wroth, C.P. 1958. On the yielding of soil. Géotechnique, 8, pp.22-52.

Rotisciani, G.M., and Miliziano, S. 2014. Guidelines for Calibration and Use of the Severn-Trent Sand Model in Modeling Cantilevered Wall-Supported Excavations. International Journal of Geomechanics, 14(6), p.04014029.

Salgado, R., Bandini, P., and Karim, A. 2000. Shear strength and stiffness of silty sand. Journal of Geo- technical and Geoenvironmental Engineering, 126(5), pp.451-462.

Shen, K., Zhang, Y., Klinkvort, R.T., Sturm, H., Jostad, H.P., Sivasithamparan, N., and Guo, Z. 2017. Numerical simulation of suction bucket under vertical tension loading. OSIG 2017, 180, pp.488-497. 
Sheng, D., Sloan, S.W., and Yu, H.S. 2000. Aspects of finite element implementation of critical state models. Computational Mechanics, 26(2), pp.185-196.

Sloan, S.W., Abbo, A.J., and Sheng, D. 2001. Refined explicit integration of elastoplastic models with automatic error control. Engineering Computations, 18(1/2), pp.121-194.

Summersgill, F.C., Kontoe, S., and Potts, D.M. 2017. On the use of nonlocal regularisation in slope stability problems. Computers and Geotechnics, 82, pp.187-200.

Taborda, D.M.G., Zdravkovic, L., Kontoe, S., and Potts, D.M 2014. Computational study on the modification of a bounding surface plasticity model for sands. Computers and Geotechnics, 59, pp.145-160.

Taiebat, M., and Dafalias, Y.F. 2008. SANISAND: Simple anisotropic sand plasticity model. International Journal for Numerical and Analytical Methods in Geomechanics, 32, pp.915-948.

Teh, K.L., Leung, C.F., Chow, Y.K., and Cassidy, M.J. 2010. Centrifuge model study of spudcan penetration in sand overlying clay. Géotechnique, 60(11), pp.825-842.

Theocharis, A.I., Vairaktaris, E., Dafalias, Y.F., and Papadimitriou, A.G. 2019. Necessary and sufficient conditions for reaching and maintaining critical state. International Journal for Numerical and Analytical Methods in Geomechanics, 43(12), pp.2041-2055.

Theocharis, A.I., Vairaktaris, E., Dafalias, Y.F., and Papadimitriou, A.G. 2017. Proof of incompleteness of critical state theory in granular mechanics and its remedy. Journal of Engineering Mechanics, 143(2), pp.1-12.

Tian, Y., and Cassidy, M.J. 2010. The challenge of numerically implementing numerous force-resultant models in the stability analysis of long on-bottom pipelines. Computers and Geotechnics, 37(1-2), pp.216-232.

Tom, J.G., O'Loughlin, C.D., White, D.J., Haghighi, A., and Maconochie, A. 2017. The effect of radial fins on the uplift resistance of buried pipelines. Géotechnique Letters, 7(1), pp.60-67.

Vaid, Y.P., Chung, E.K.F., and Kuerbis, R.H. 1990. Stress path and steady state. Canadian Geotechnical Journal, 27(1), pp.1-7.

Vaid, Y.P., Sivathayalan, S., and Stedman, D. 1999. Influence of specimen reconstitution method on the undrained response and microstructure of silty sand. Geotechnical Testing Journal, 22(3), pp.187-195.

White, D.J., Teh, K.L., Leung, C.F., and Chow, Y.K. 2008. A comparison of the bearing capacity of flat and 
conical circular foundations on sand. Géotechnique, 58(10), pp.781-792.

Yamashita, S., Jamiolkowski, M., and Lo Presti, D.C.F. 2000. Stiffness nonlinearity of three sands. Journal of Geo- technical and Geoenvironmental Engineering, 126(October), pp.929-938.

Yang, Z., Elgamal, A., and Parra, E. 2003. Computational Model for Cyclic Mobility and Associated Shear Deformation. Journal of Geotechnical and Geoenvironmental Engineering, 129(12), pp.1119-1127.

Yimsiri, S., and Soga, K. 2010. Dem analysis of soil fabric effects on behaviour of sand. Geotechnique, 60(6), pp.483-495.

Yoshimine, M., Ishihara, K., and Vargas, W. 1998. effects of principal stress direction and intermediate principal stress. Soils and Foundations, 38(3), pp.179-188.

Ziotopoulou, K., and Boulanger, R.W. 2013. Calibration and implementation of a sand plasticity plane-strain model for earthquake engineering applications. Soil Dynamics and Earthquake Engineering, 53, pp.268280. 


\section{Tables captions}

Table 1: Parameters with the governing equations and calibration procedure

Table 2: Physical properties of sands

Table 3 : Model parameters for Erksak and UWA silica sand

Table 4 : UWA silica sand model parameters obtained using optimisation and from individual tests

\section{Figure captions}

Fig. 1: Schematic diagram of the position of bounding surface, dilatancy surface and critical state surface with respect to the yield surface in the $q-p^{\prime}$ space (modified after Dafalias and Manzari 2004)

Fig. 2 : Calibration of the CSL line for (a) UWA Silica sand (b) Erksak Sand

Fig. 3: Variation of stress ratio at peak stress with state parameter for (a) UWA Silica Sand (b) Erksak sand

Fig. 4: Variation of stress ratio at phase transformation point with state parameter for (a) UWA Silica Sand (b) Erksak sand

Fig. 5: Determining $G_{0}$ by matching the initial slopes of the $q-\varepsilon_{a}$ response in a drained TXC test in UWA silica sand

Fig. 6: Setting the initial value of $h_{\text {in }}$ to be used for UWA Silica sand

Fig. 7: Initial approximation of $A_{0}$ from measured drained TXC data before optimisation for (a) UWA Silica sand (b) Erksak sand

Fig. 8: Calculation of error for objective function

Fig. 9: Comparison of measured and simulated responses for triaxial compression tests on Erksak sand in (a), (b), (c) drained condition (d) undrained condition

Fig. 10 : Comparison of measured and simulated responses for triaxial compression tests on UWA silica sand in (a), (b) drained condition (c) undrained condition 
Fig. 11: Demonstration of the effectiveness of the optimisation approach against a new set of triaxial responses

Fig. 12: Comparison of measured and simulated responses of triaxial extension tests in UWA silica sand

Fig. 13: Comparison of simulated responses with constant $b$ and varying fabric inclination angle $\beta$ (a) at small shear strain levels and (b) at large strain levels

Fig. 14: Comparison of measured and simulated undrained simple shear responses on anisotropically consolidated samples

Fig. 15 : Representative finite element mesh for (a) footing problem (b) anchor problem at $H / D=3$

Fig. 16: Comparison of simulated and measured mobilised $\mathrm{N}_{\gamma}$ for circular footings in UWA silica sand in (a) medium dense sand $\left(R_{D}=54 \%\right)$ (b) dense sand $\left(R_{D}=78 \%\right)$

Fig. 17: Effect of varying $G_{0}$ and $h_{i n}$ on simulations for the case of a circular footing in UWA sand at $R_{D}=$ $78 \%$

Fig. 18: Measured and simulated stiffness response compared with elastic theory for the case of a circular footing in UWA sand at $R_{D}=78 \%$

Fig. 19 : Comparison of simulated and measured mobilised $\mathrm{N}_{\gamma}$ for circular anchors in UWA silica sand at $H / D=3, R_{D}=85 \%$ 


\section{Figures}

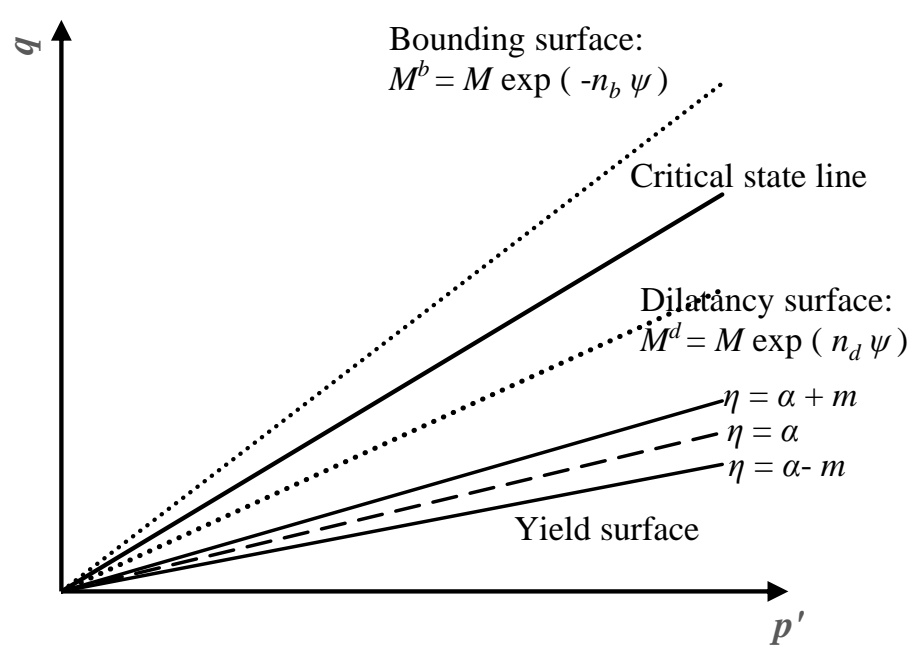

Fig. 1 : Schematic diagram of the position of bounding surface, dilatancy surface and critical state surface with respect to the yield surface in the $q-p^{\prime}$ space (modified after Dafalias and Manzari 2004) 


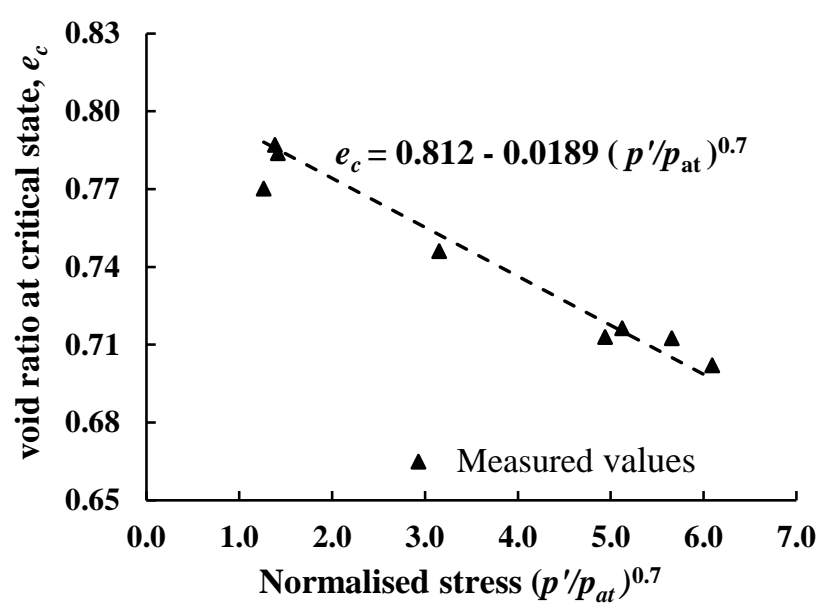

(a)

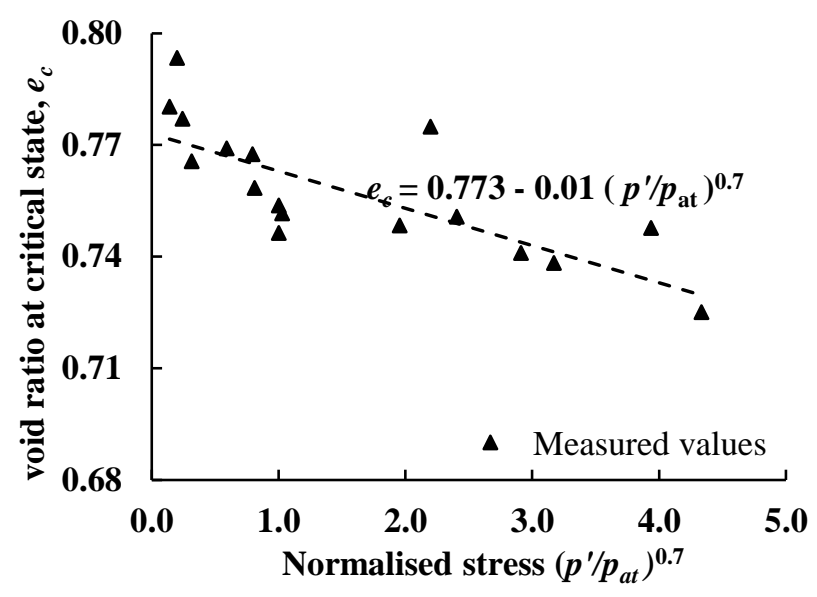

(b)

Fig. 2 : Calibration of the CSL line for (a) UWA Silica sand (b) Erksak Sand 


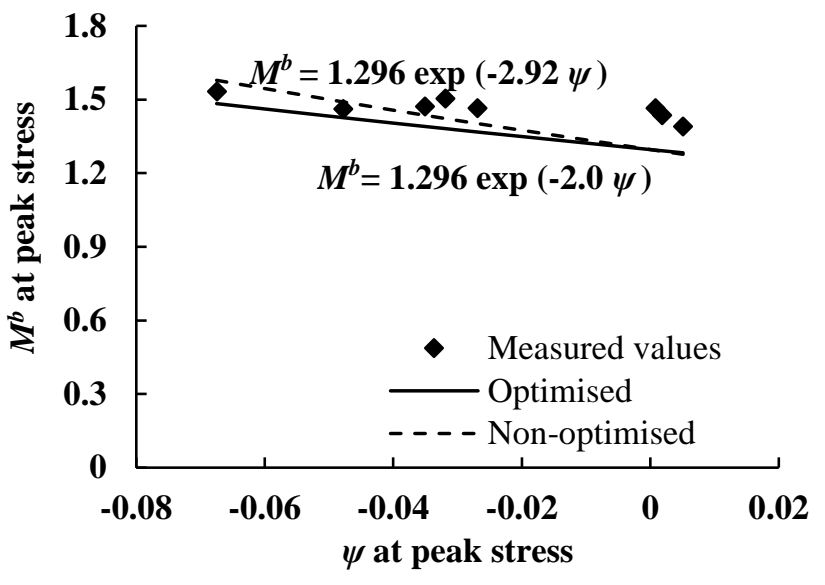

(a)

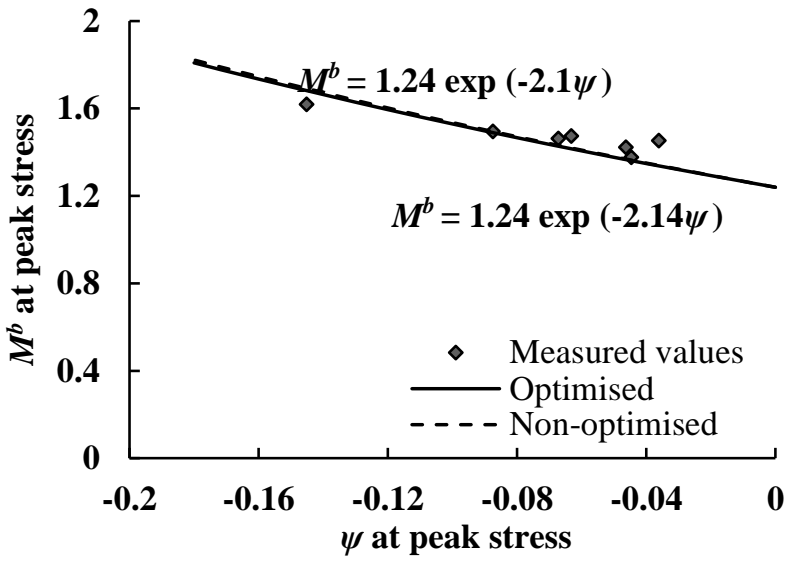

(b)

Fig. 3: Variation of stress ratio at peak stress with state parameter for (a) UWA Silica Sand (b) Erksak sand 


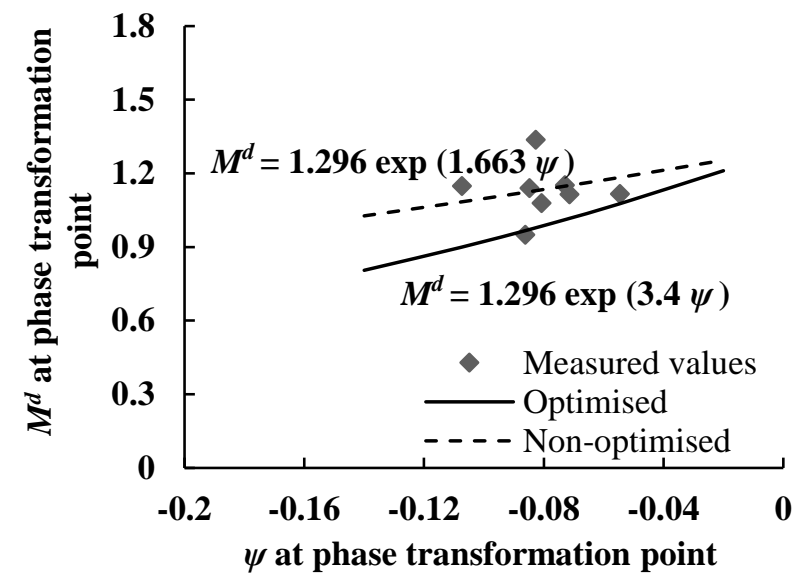

(a)

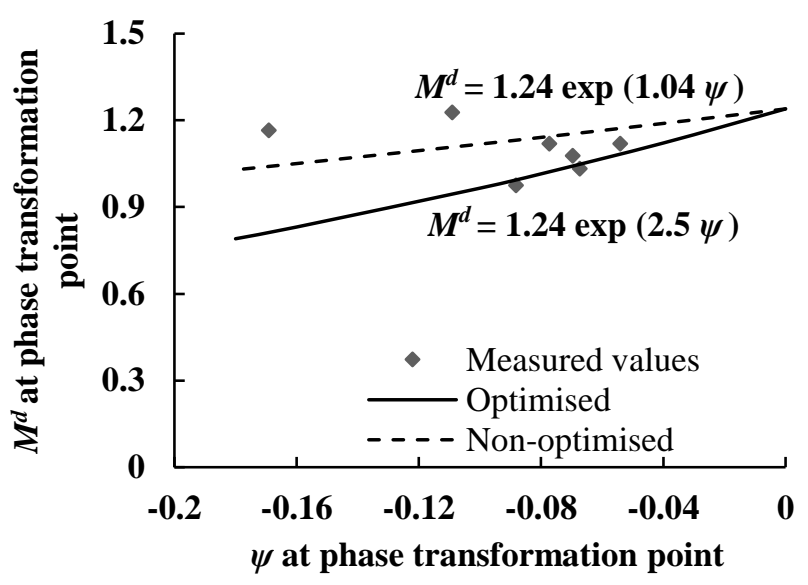

(b)

Fig. 4: Variation of stress ratio at phase transformation point with state parameter for (a) UWA Silica Sand (b) Erksak sand 


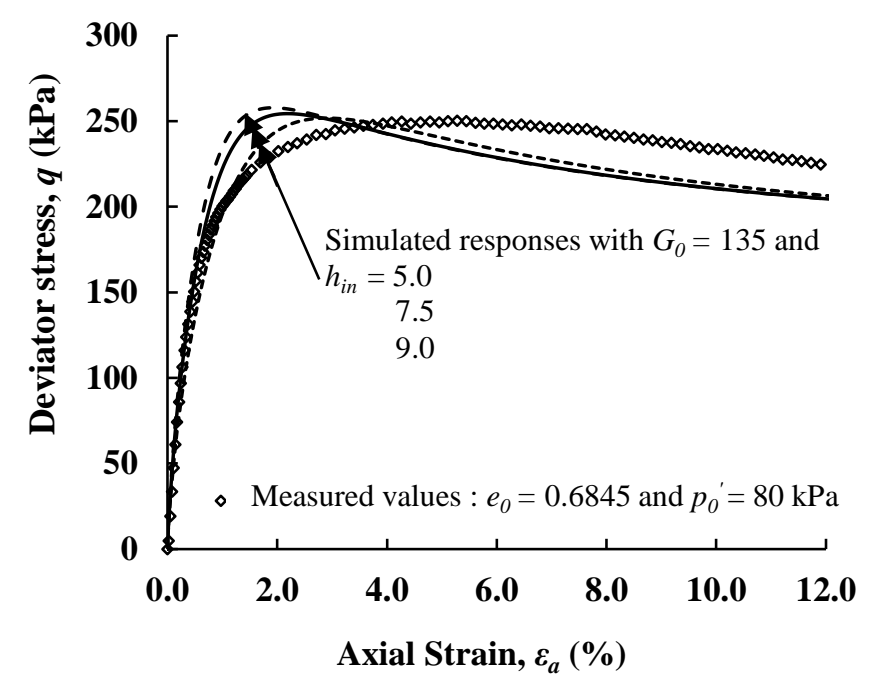

Fig. 5: Determining $G_{0}$ by matching the initial slopes of the $q-\varepsilon_{a}$ response in a drained TXC test in UWA silica sand 


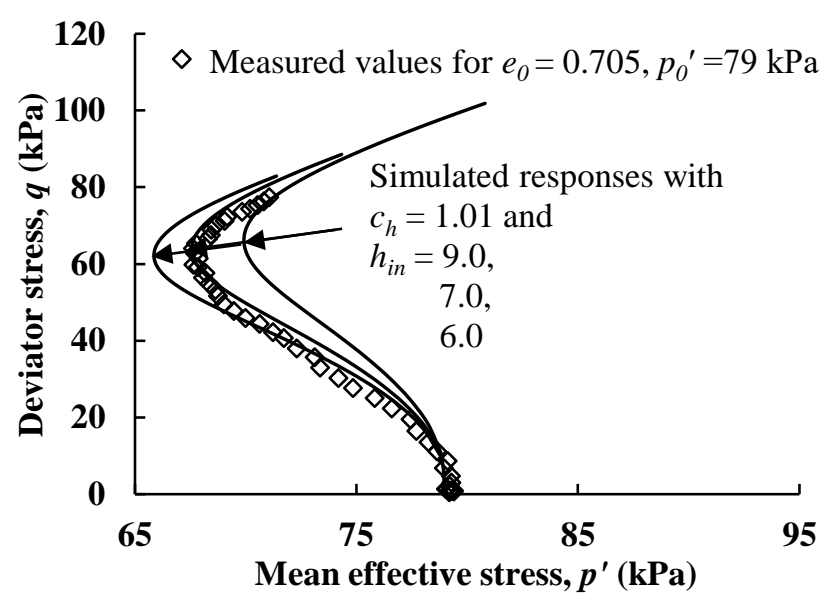

Fig. 6: Setting the initial value of $h_{i n}$ to be used for UWA Silica sand 

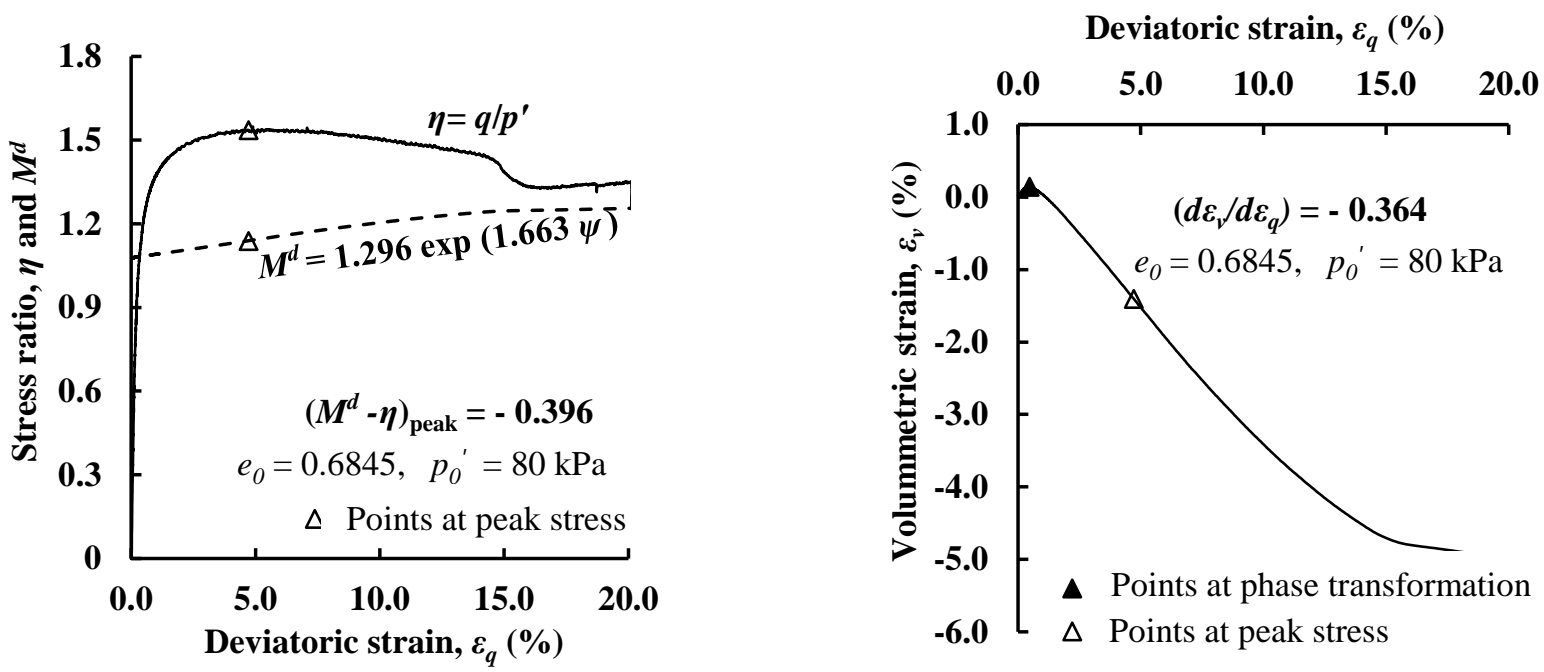

(a)
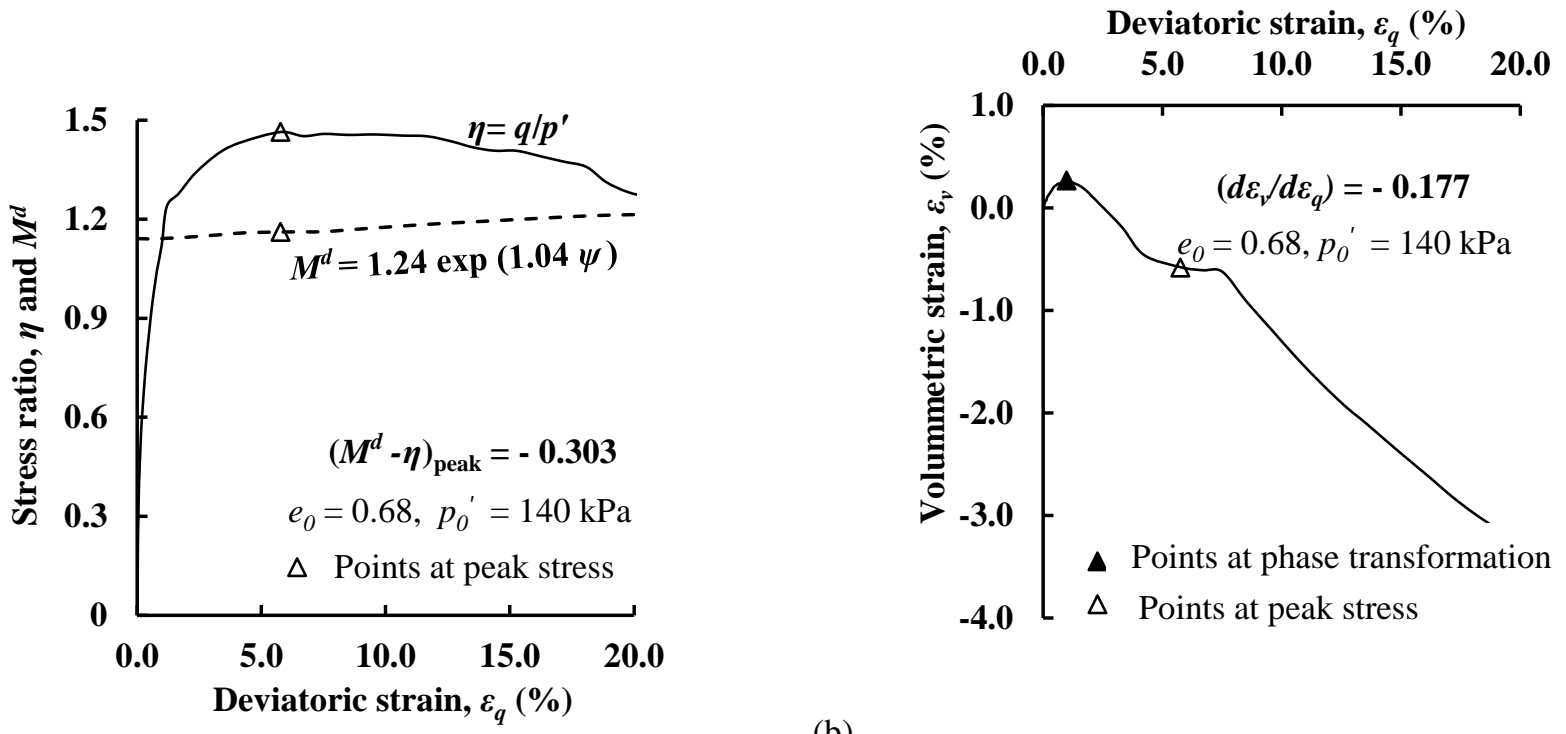

(b)

Fig. 7: Initial approximation of $A_{0}$ from measured drained TXC data before optimisation for (a) UWA Silica sand (b) Erksak sand 


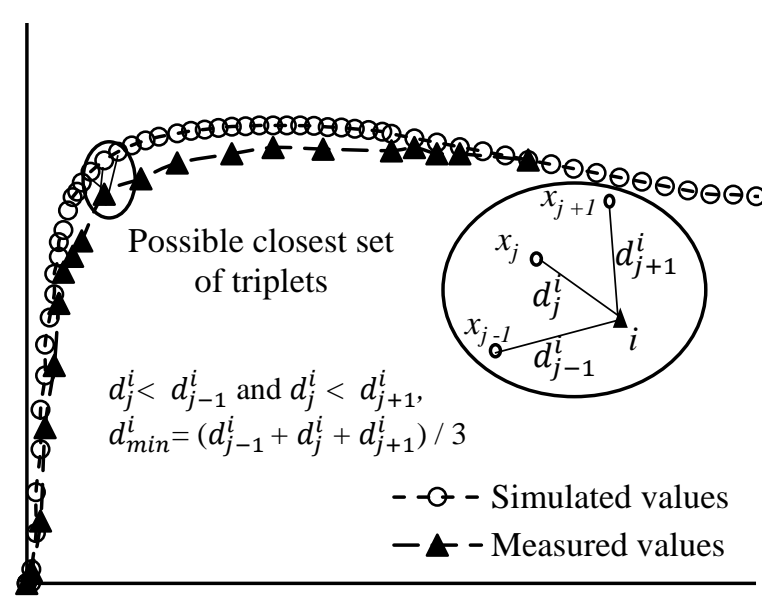

Fig. 8: Calculation of error for objective function 

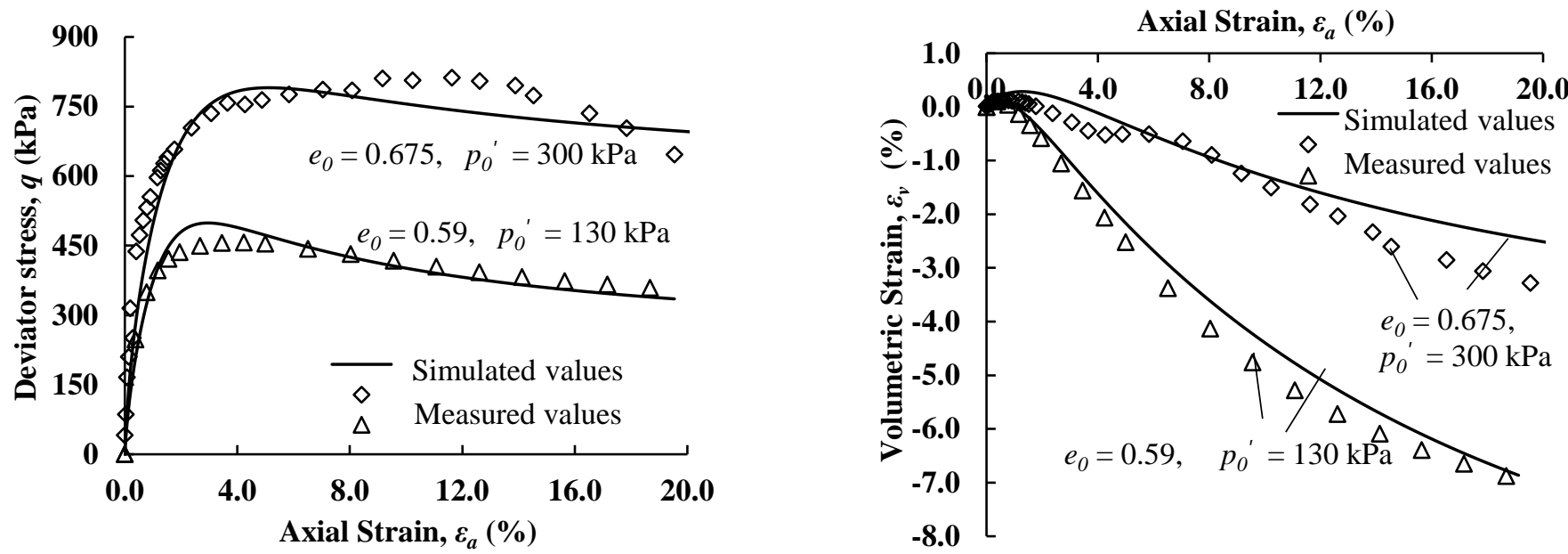

(a)
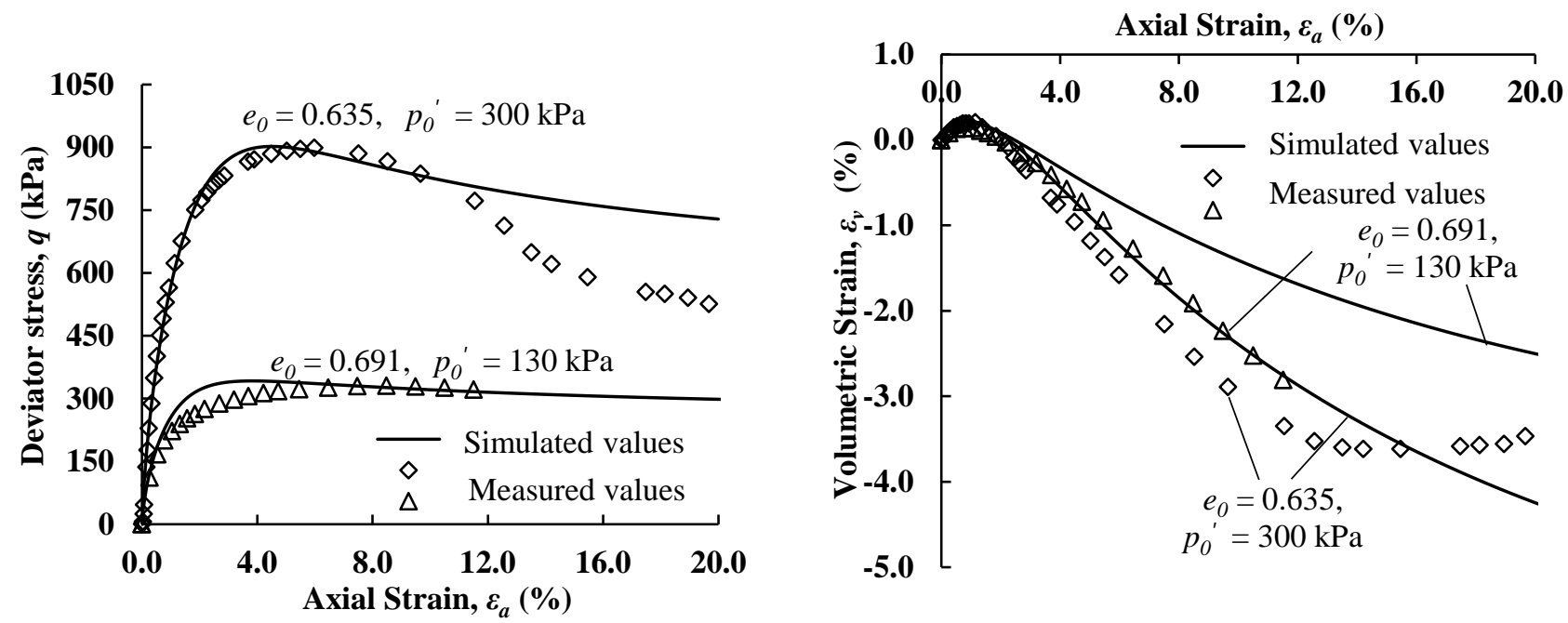

(b)
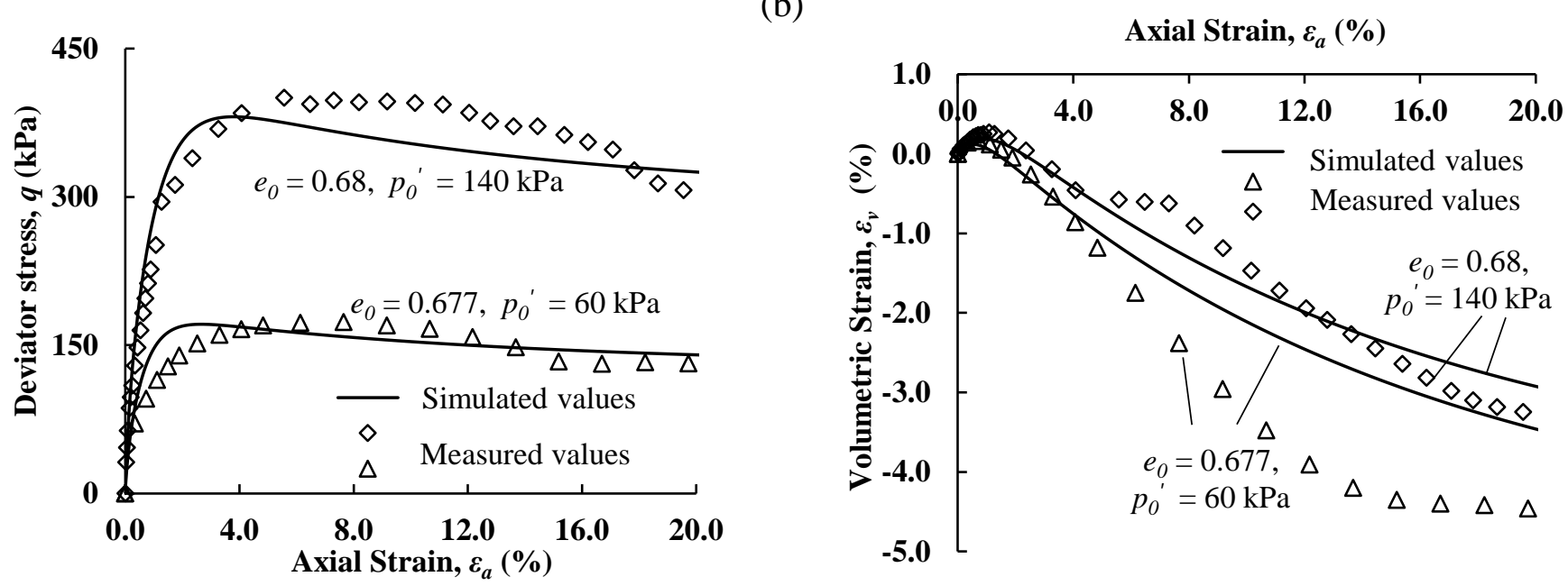

(c) 

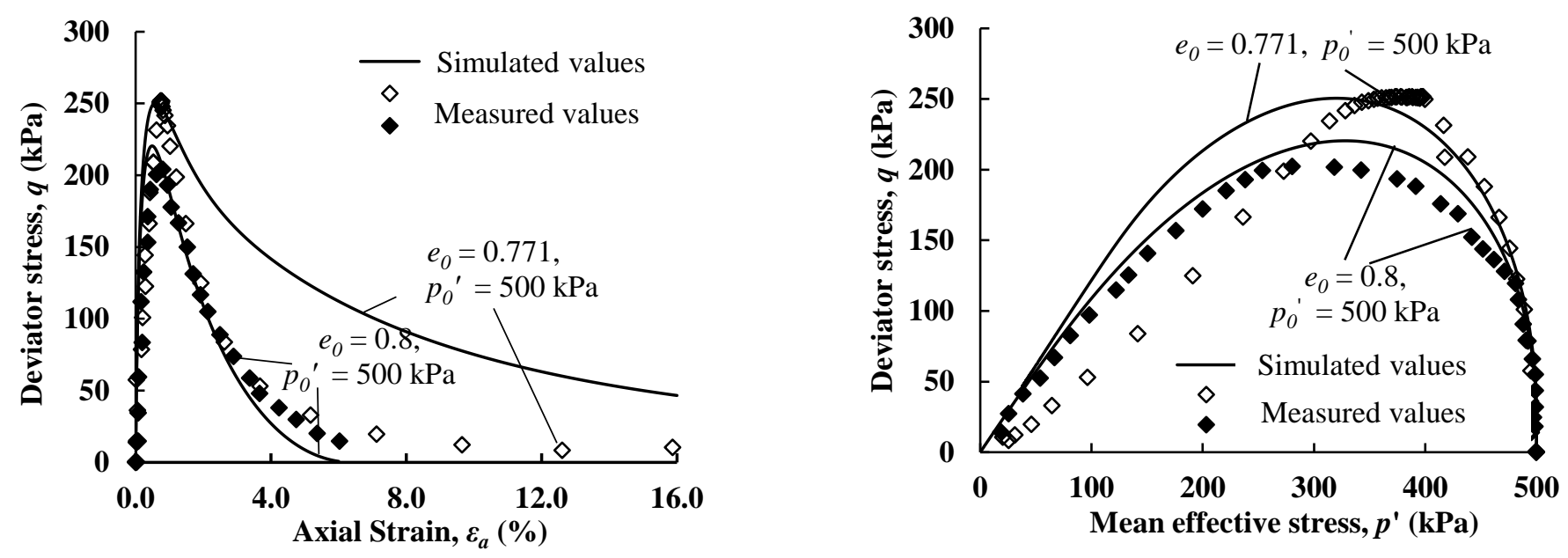

(d)

Fig. 9: Comparison of measured and simulated responses for triaxial compression tests on Erksak sand in (a), (b), (c) drained condition (d) undrained condition 

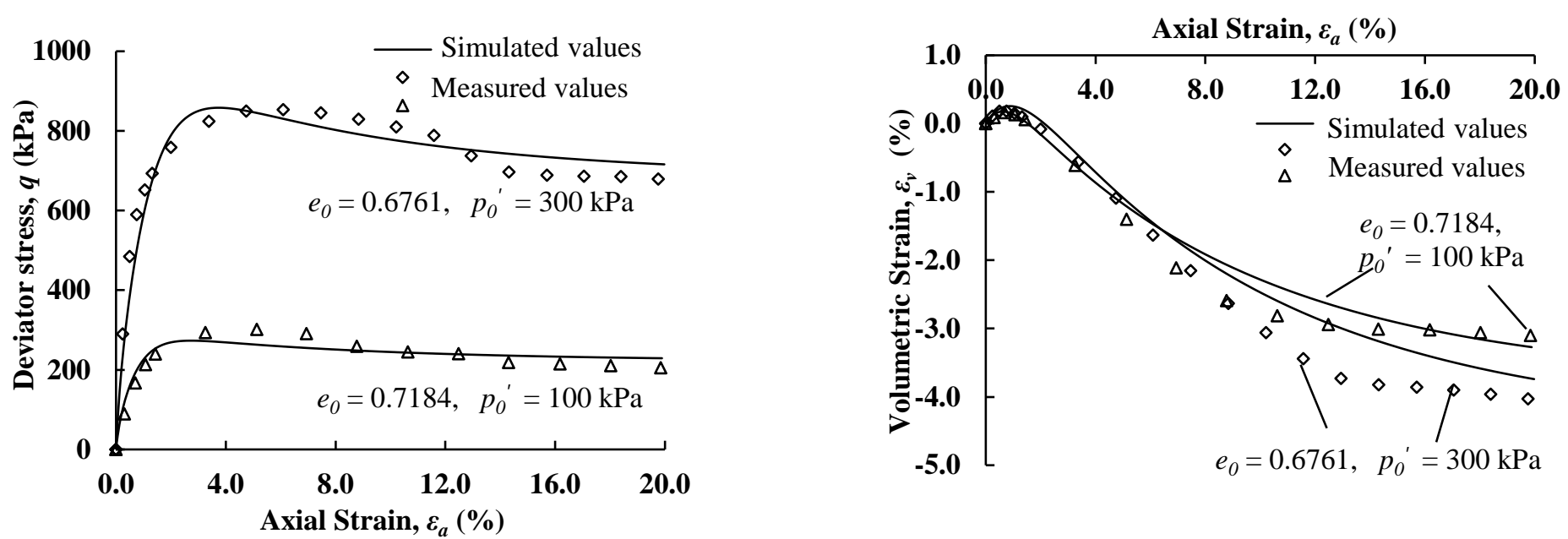

(a)
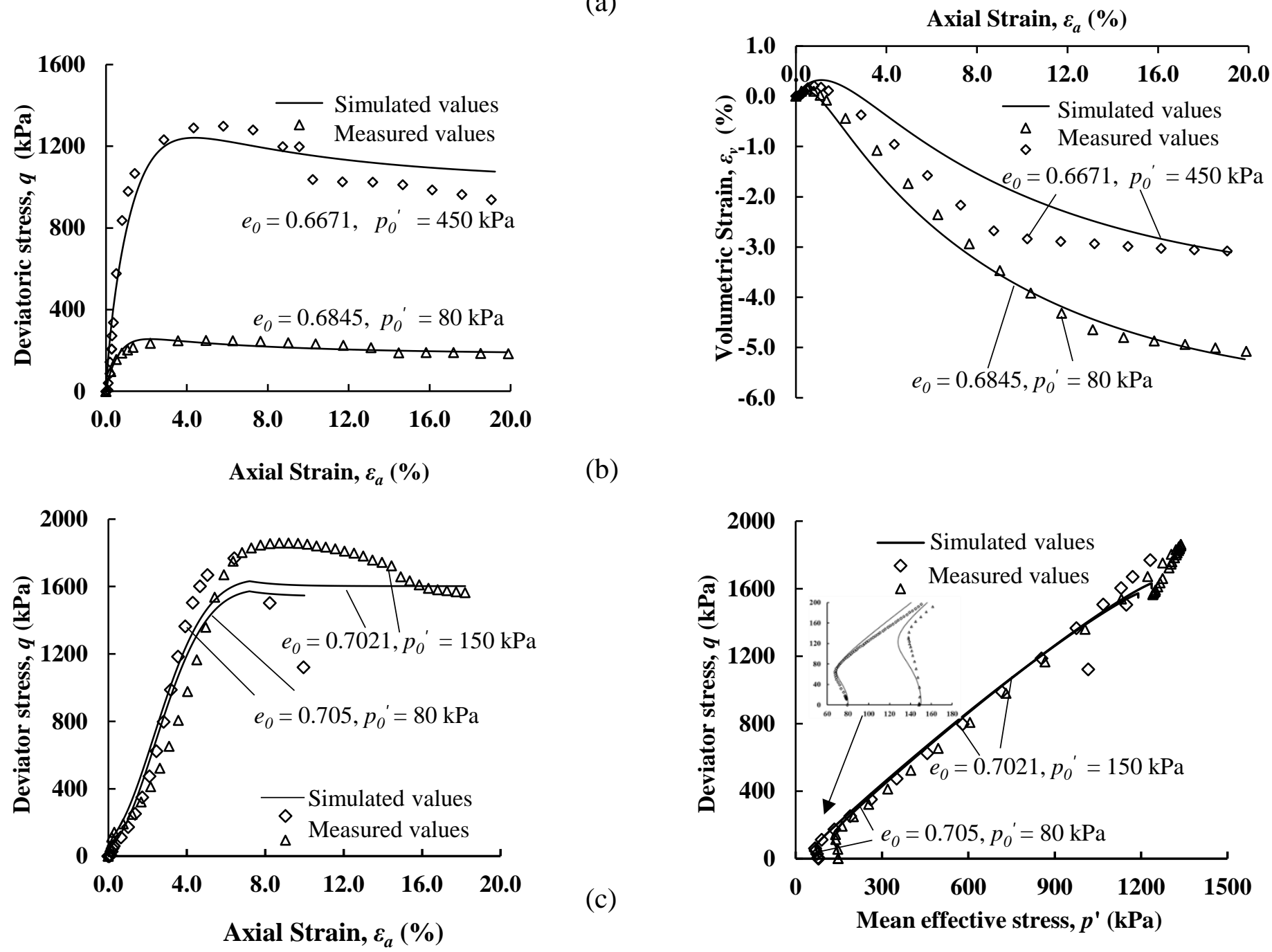

(b)

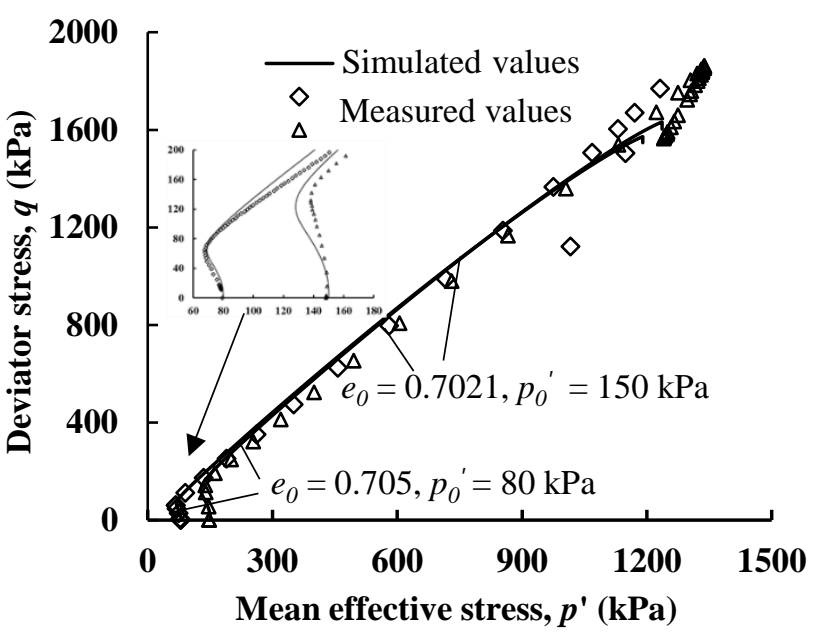

Fig. 10 : Comparison of measured and simulated responses for triaxial compression tests on UWA silica sand in (a), (b) drained condition (c) undrained condition 

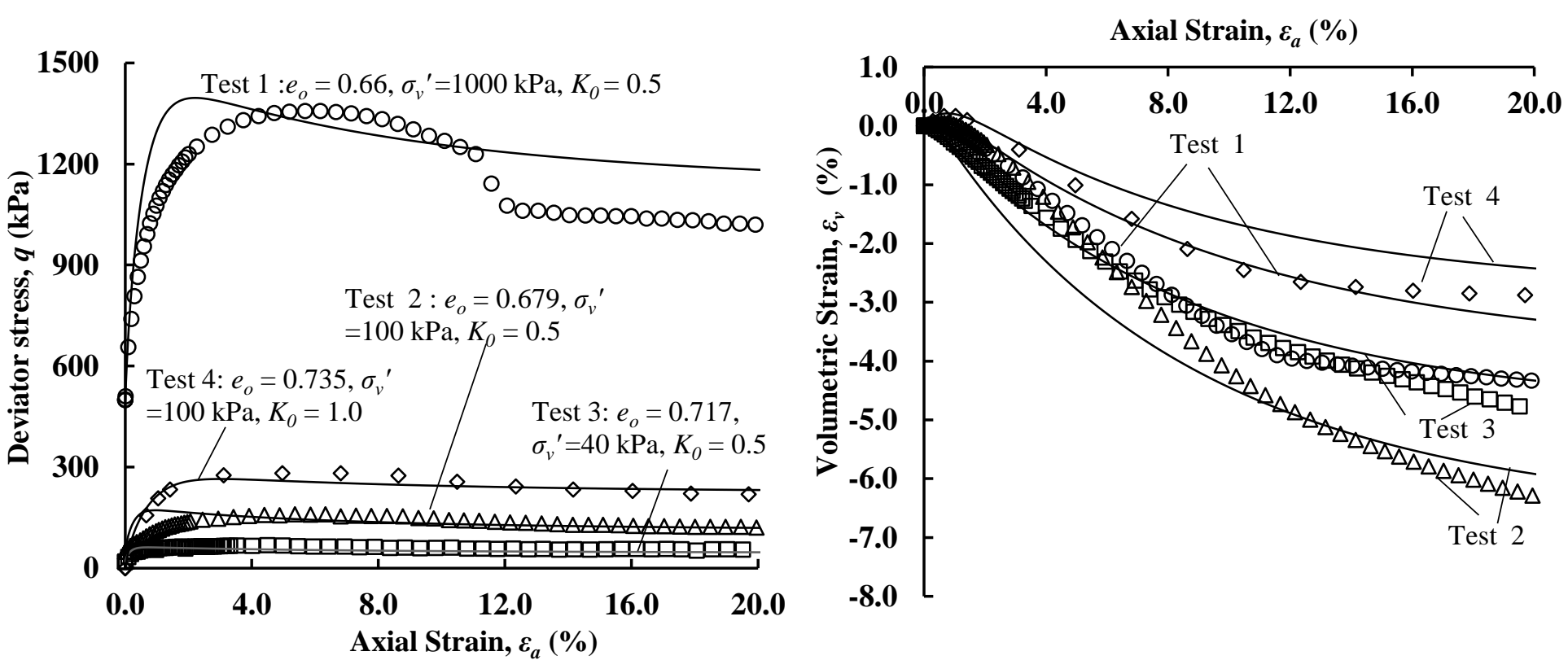

Fig. 11: Demonstration of the effectiveness of the optimisation approach against a new set of triaxial responses 

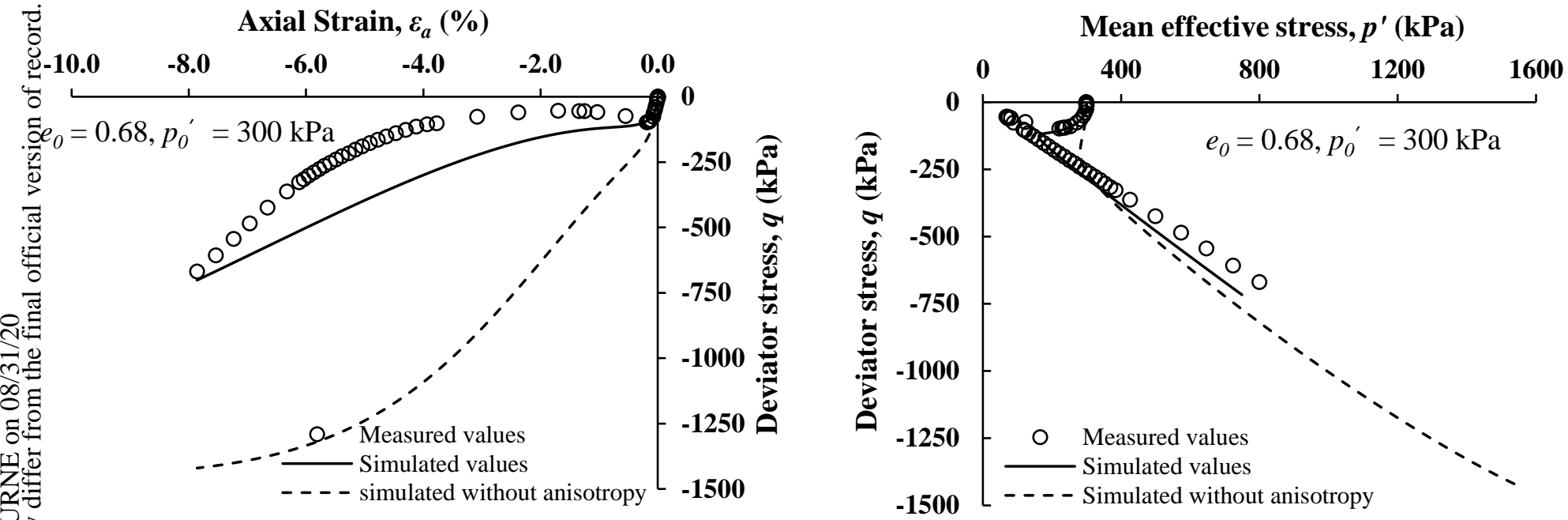

Axial Strain, $\varepsilon_{a}(\%)$
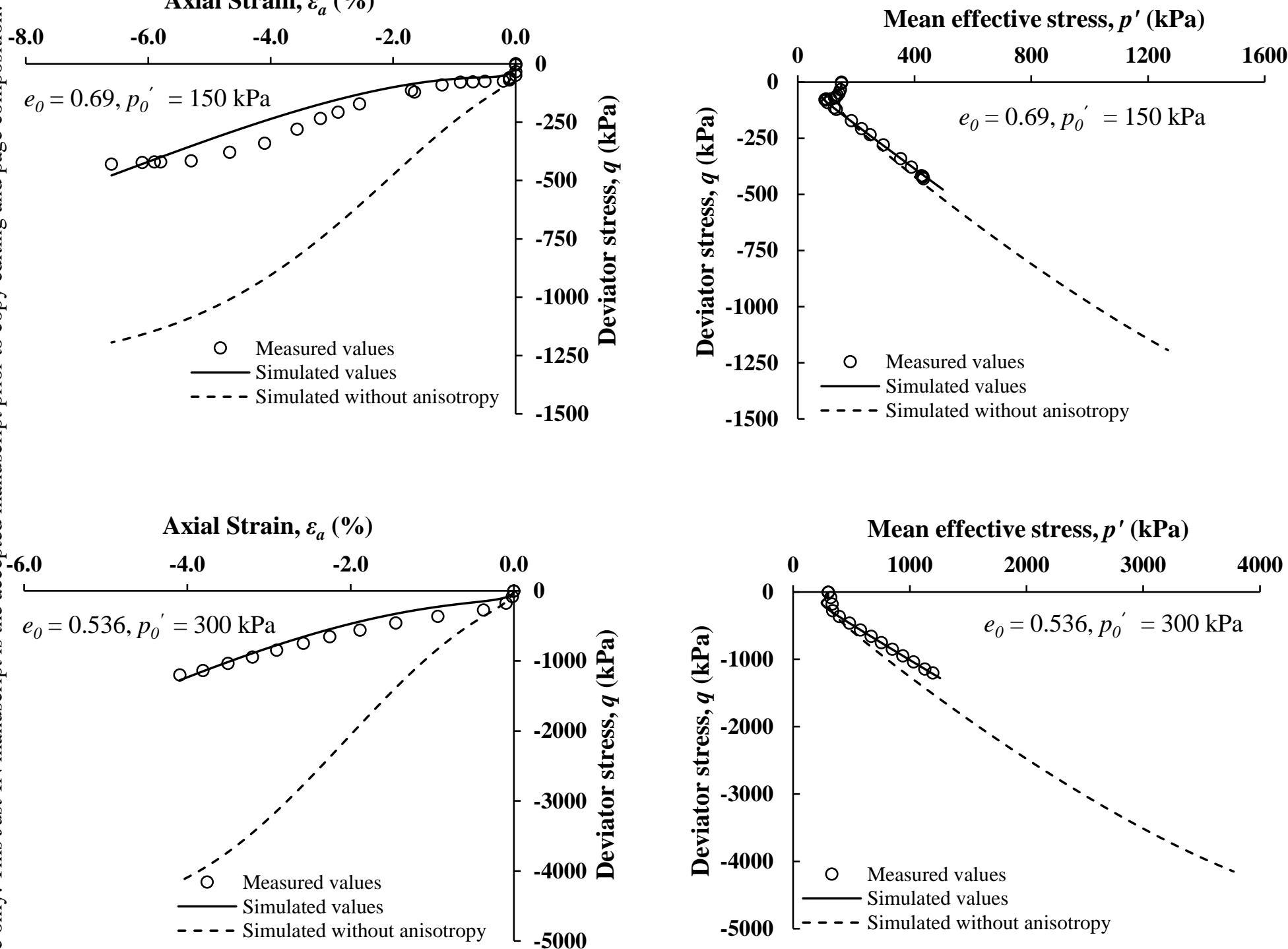

Fig. 12: Comparison of measured and simulated responses of triaxial extension tests in UWA silica sand 


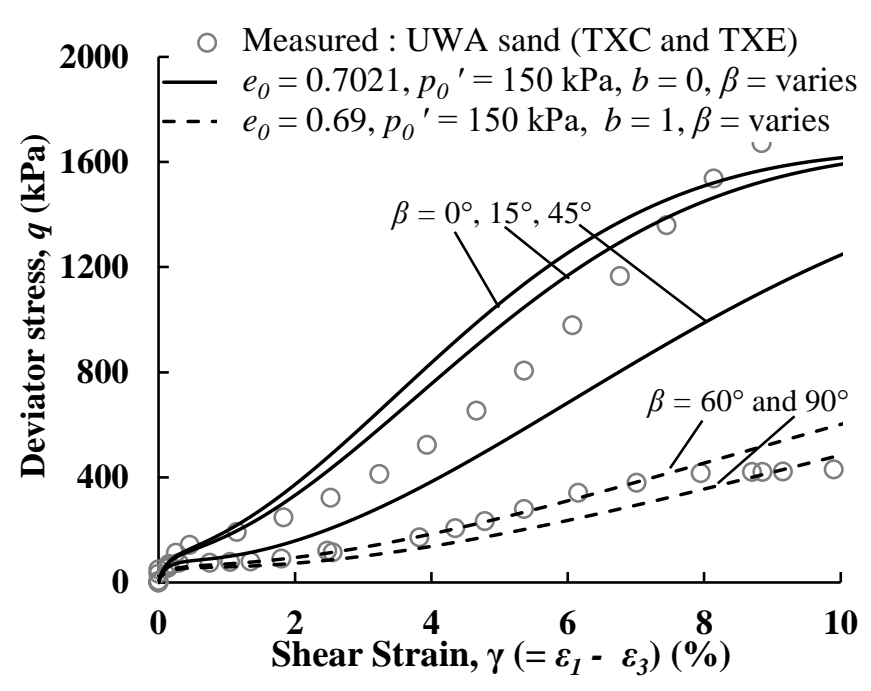

(a)

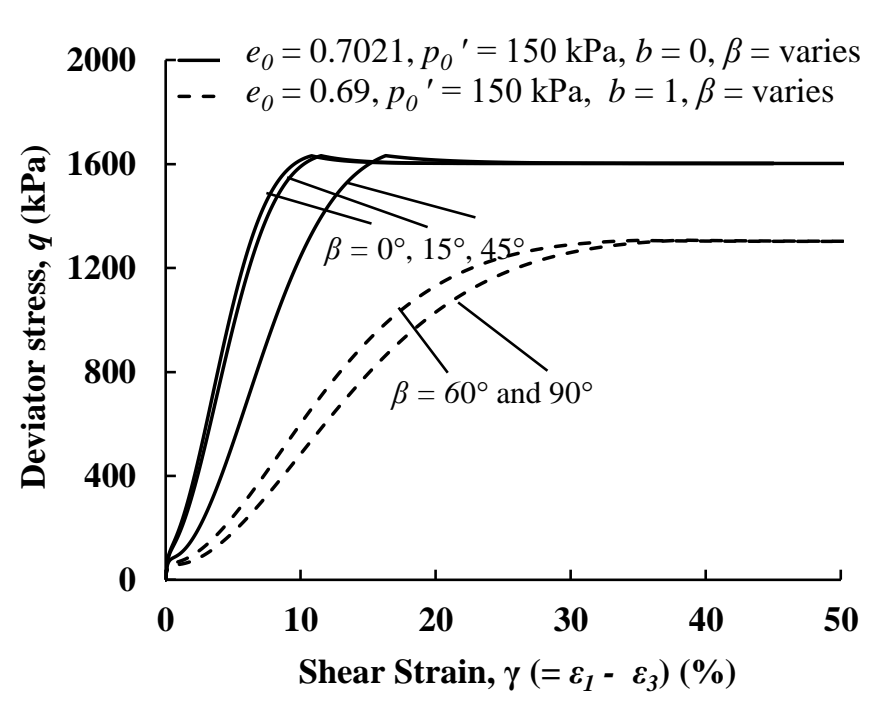

(b)

Fig. 13: Comparison of simulated responses with constant $b$ and varying fabric inclination angle $\beta$ (a) at small shear strain levels and (b) at large strain levels 


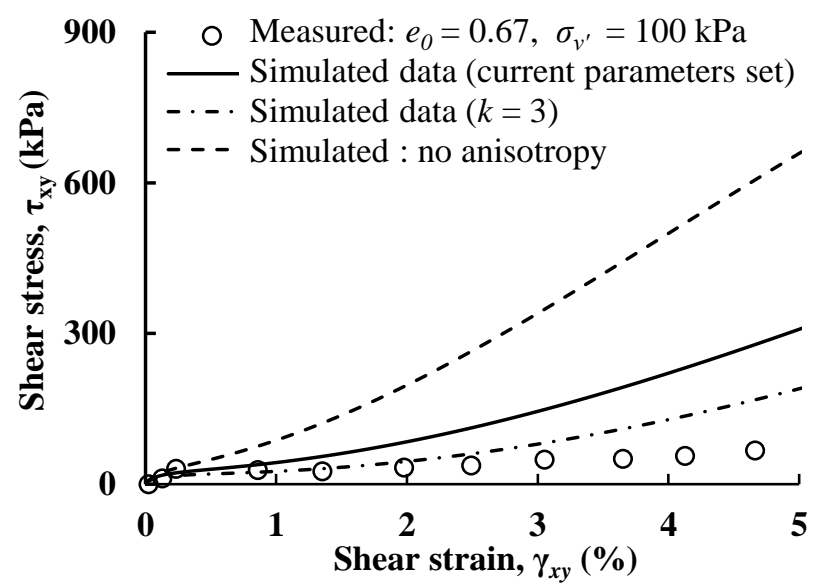

Fig. 14: Comparison of measured and simulated undrained simple shear responses on anisotropically consolidated samples 


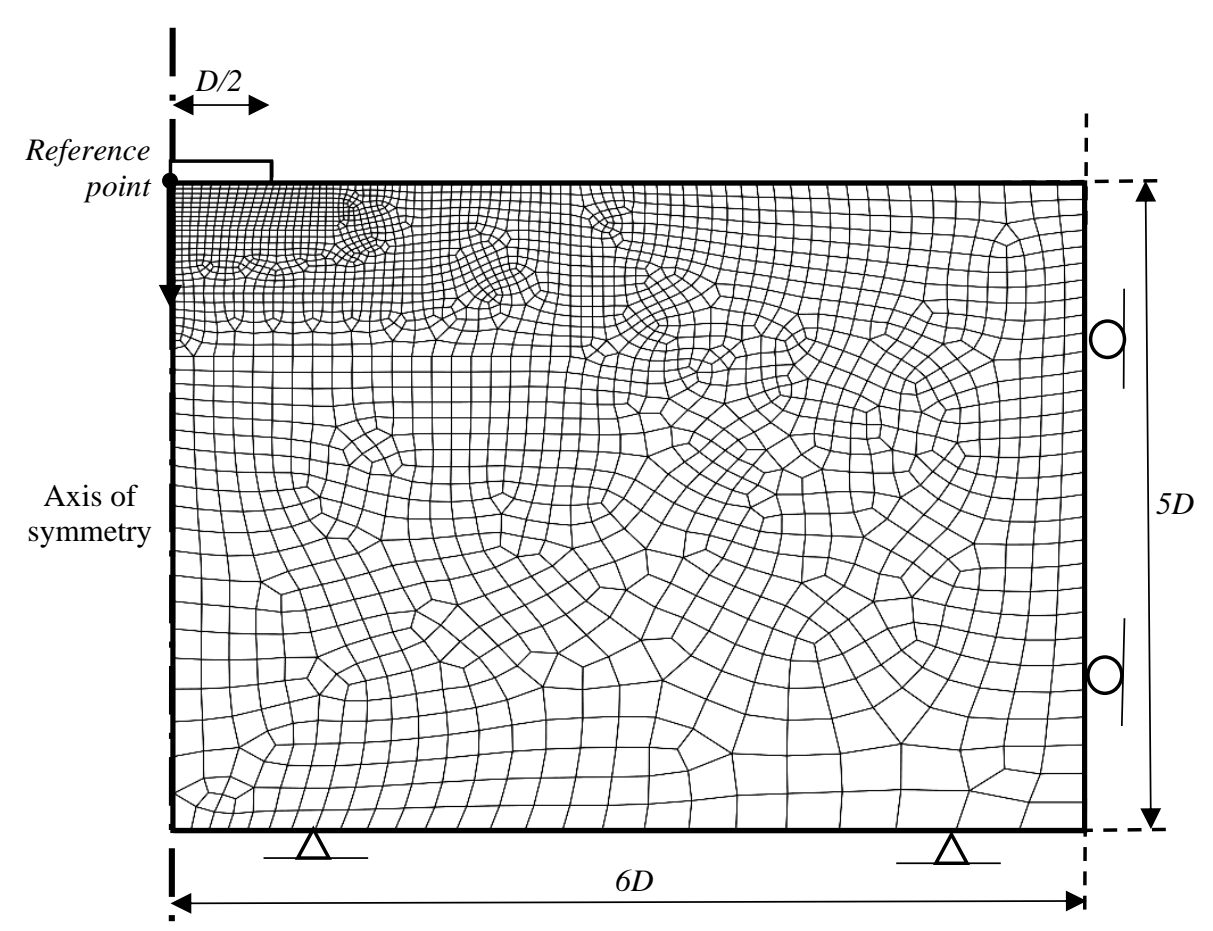

(a)

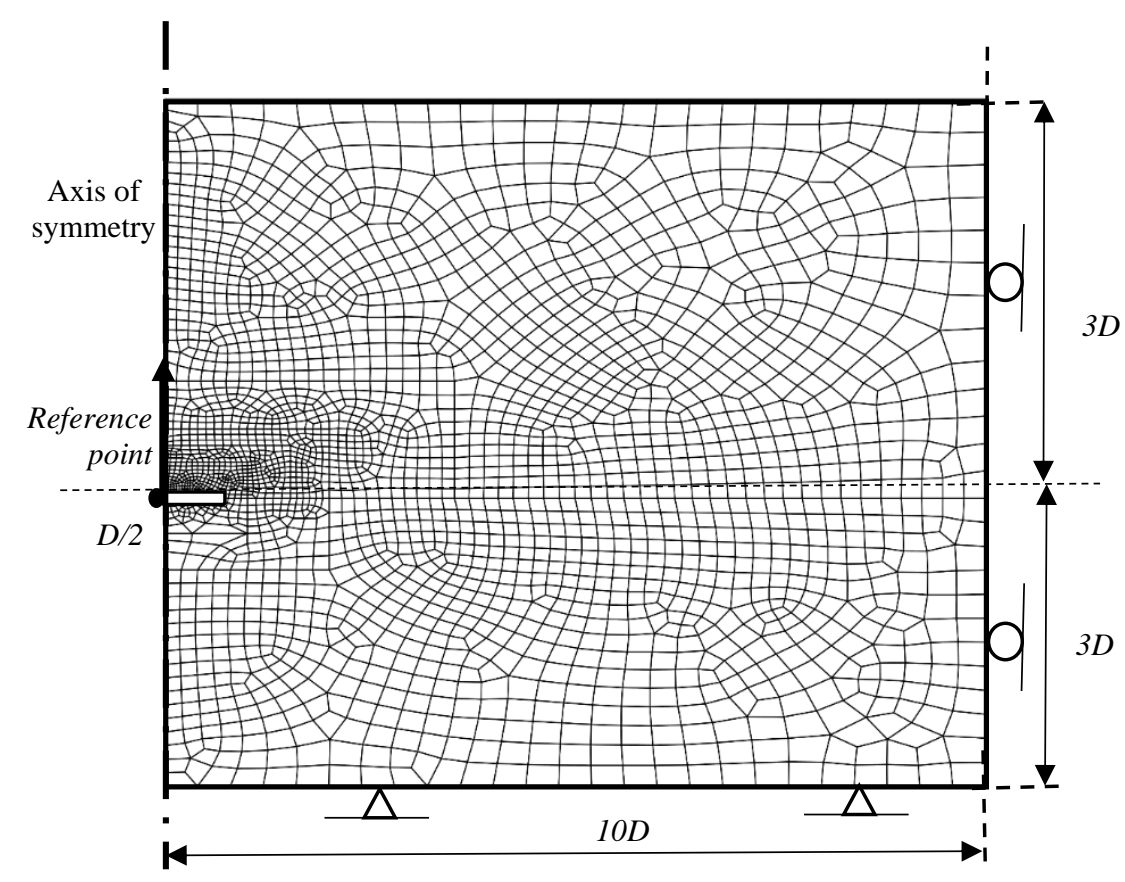

(b)

Fig. 15 : Representative finite element mesh for (a) footing problem (b) anchor problem at $H / D=3$ 


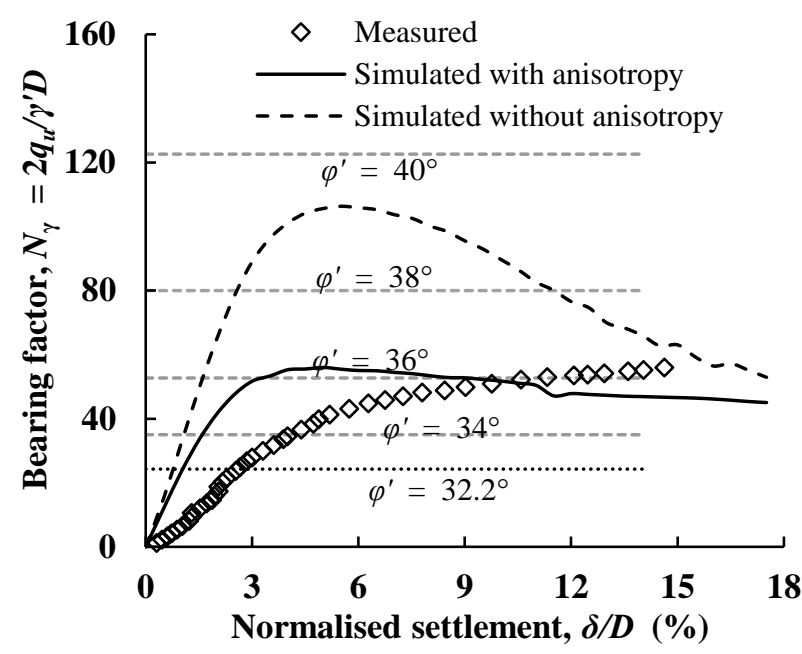

(a)

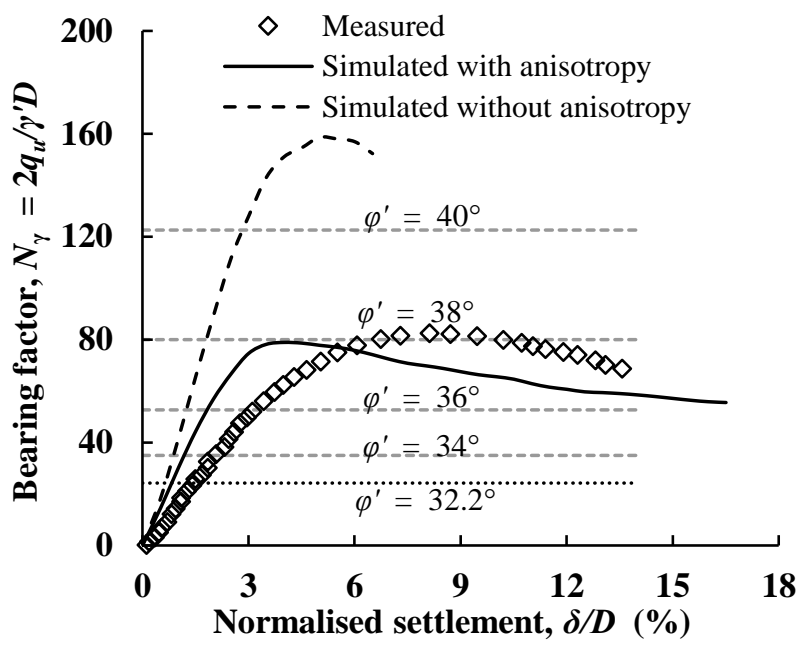

(b)

Fig. 16: Comparison of simulated and measured mobilised $N_{\gamma}$ for circular footings in UWA silica sand in (a) medium dense sand $\left(R_{D}=54 \%\right)$ (b) dense sand $\left(R_{D}=78 \%\right)$ 


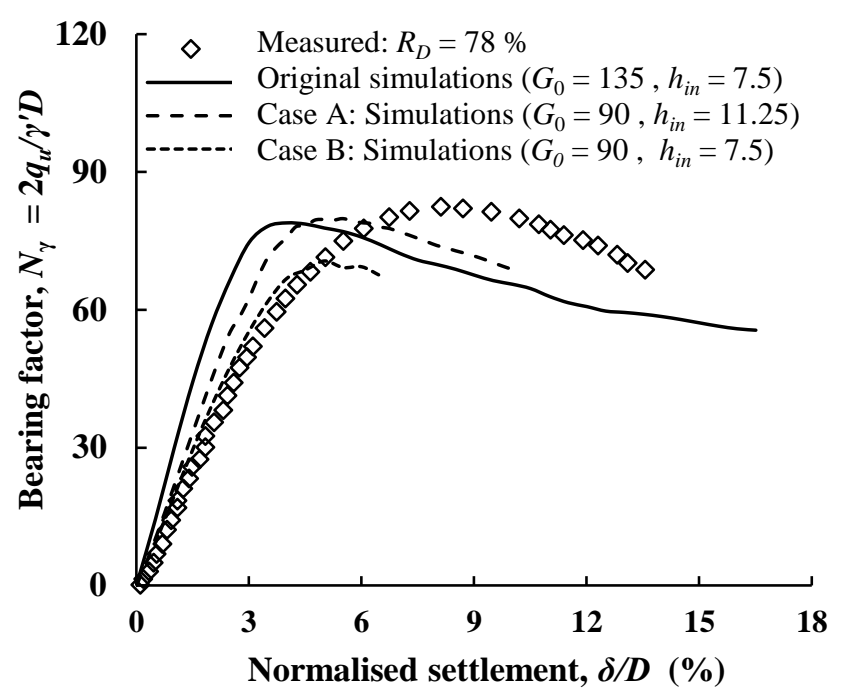

Fig. 17: Effect of varying $G_{0}$ and $h_{i n}$ on simulations for the case of a circular footing in UWA sand at $R_{D}$ $=78 \%$ 


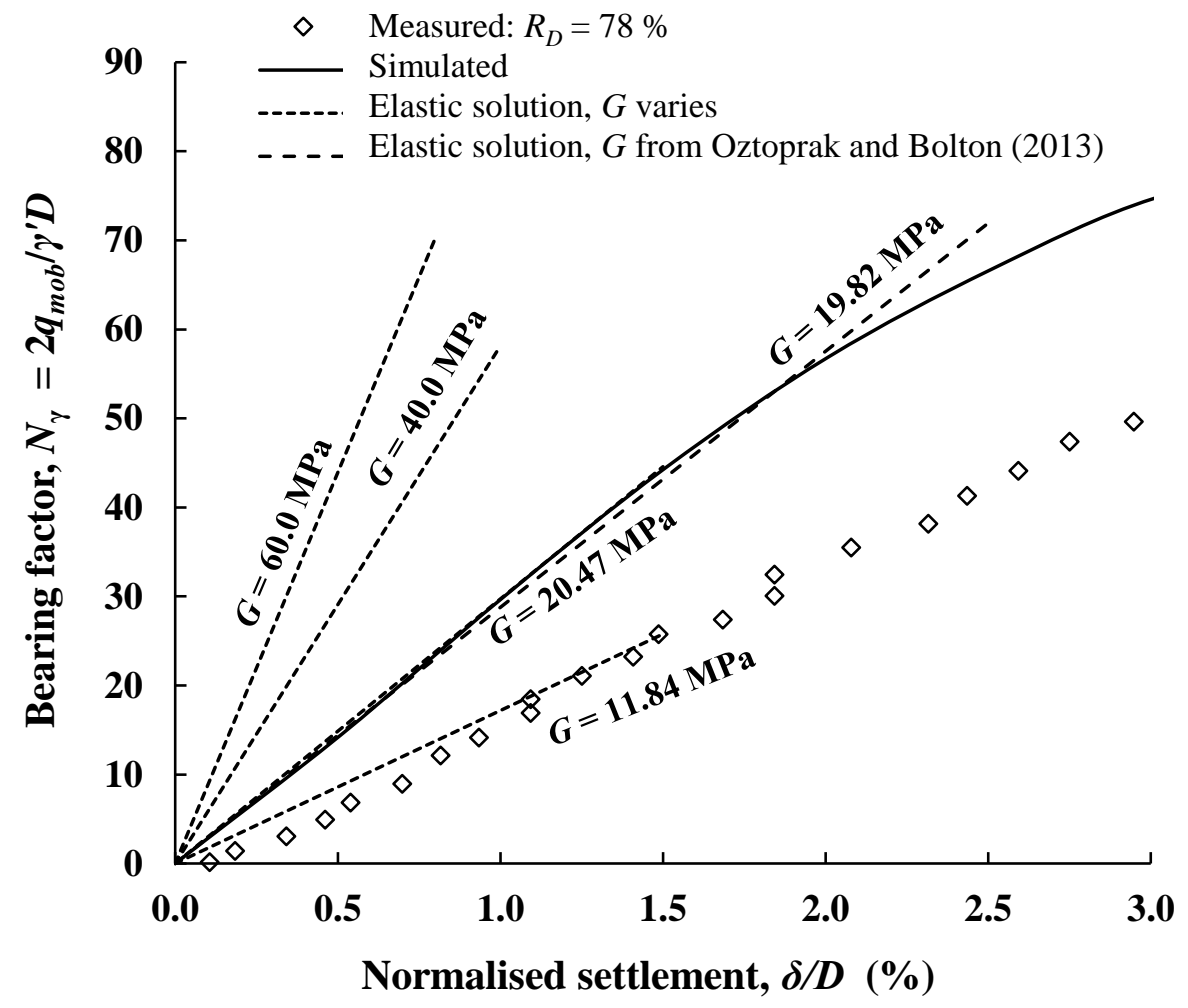

Fig. 18: Measured and simulated stiffness response compared with elastic theory for the case of a circular footing in UWA sand at $R_{D}=78 \%$ 


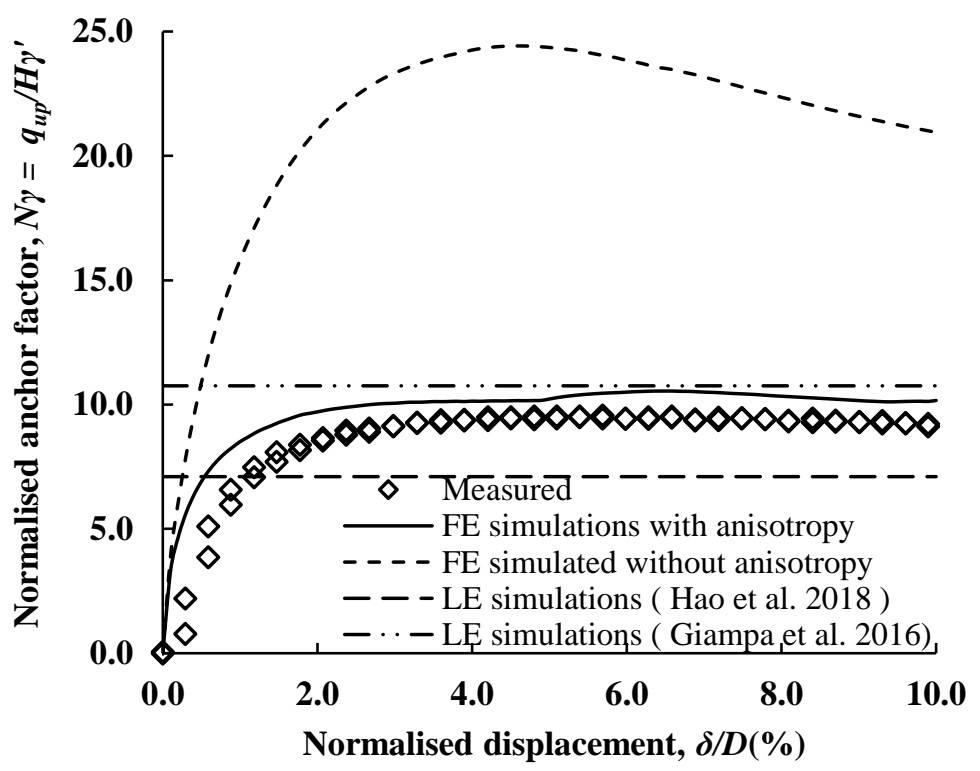

Fig. 19 : Comparison of simulated and measured mobilised $N_{\gamma}$ for circular anchors in UWA silica sand at $H / D=3, R_{D}=85 \%$ 
Table 1: Parameters with the governing equations and calibration procedure

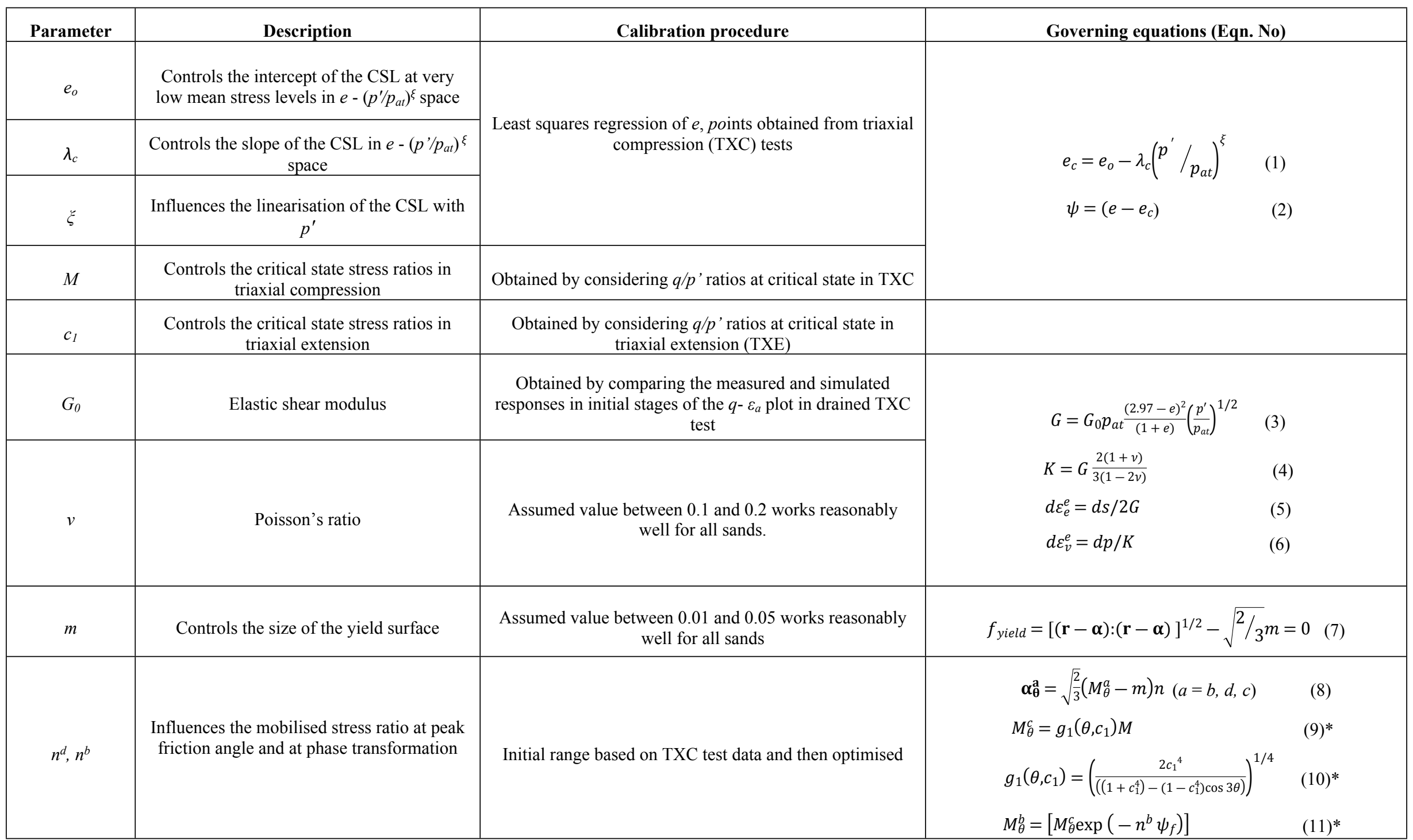




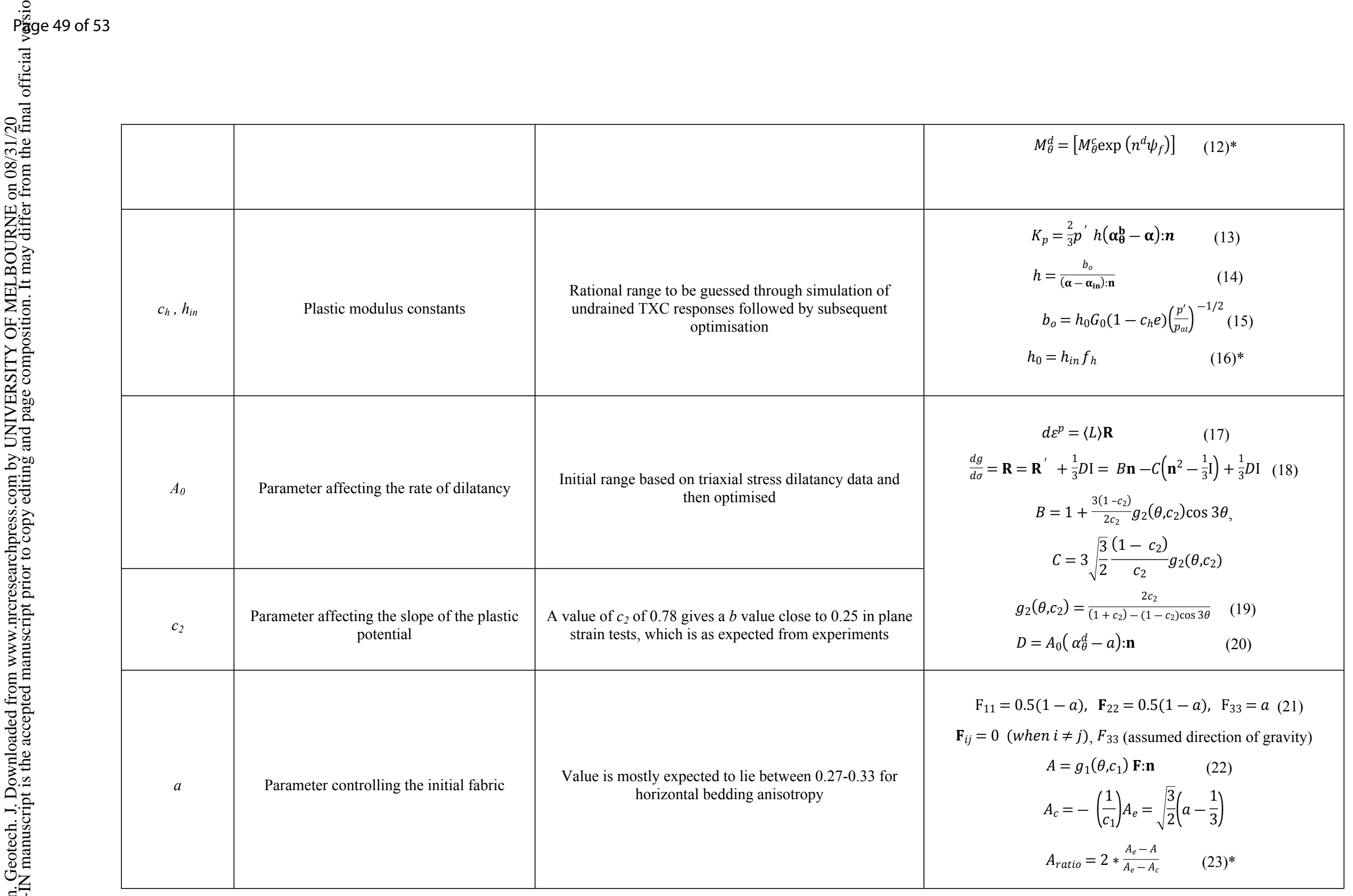




\begin{tabular}{|c|c|c|c|}
\hline$b_{l}, b_{2}, k$ & $\begin{array}{l}\text { Factors to scale the state parameter and } \\
\text { plastic modulus for loading conditions other } \\
\text { than triaxial compression }\end{array}$ & Trial and error on undrained TXE data & $\begin{array}{c}f=\frac{1}{b_{1}+b_{2}\left(A_{\text {ratio }}\right)^{b_{3}}} \quad(24)^{*} \\
b_{3}=\frac{\log \left(\frac{1-b_{1}}{b_{2}}\right)}{\log 2} \\
f_{h}=f^{k} \quad(25)^{*} \\
\psi_{f}=f \psi \quad(26)^{*}\end{array}$ \\
\hline
\end{tabular}

Note: *Additional features introduced to the original model 
Table 2: Physical properties of sands

\begin{tabular}{ccc}
\hline Property & Erksak Sand 330/.7 a & UWA Silica sand \\
Angularity & Rounded to Sub rounded & Sub-rounded to sub-angular \\
Specific gravity $\left(G_{s}\right)$ & 2.66 & 2.67 \\
Maximum void ratio $\left(e_{\max }\right)$ & 0.753 & 0.789 \\
Minimum void ratio $\left(e_{\min }\right)$ & 0.527 & 0.512 \\
Uniformity coefficient $\left(C_{U}\right)$ & 1.8 & 1.73 \\
Particle mean size, $\left(d_{50}\right)$ & $0.33 \mathrm{~mm}$ & $0.2 \mathrm{~mm}$ \\
Sample preparation technique & Wet pluviation & Wet pluviation \\
\hline
\end{tabular}

${ }^{\mathrm{a}}$ Been et al. (1991) 
Table 3 : Model parameters for Erksak and UWA silica sand

\begin{tabular}{|c|c|c|c|}
\hline Model parameters & Symbol & $\begin{array}{c}\text { Erksak Sand } \\
\mathbf{3 3 0} / .7 \\
\end{array}$ & $\begin{array}{c}\text { UWA } \\
\text { silica sand }\end{array}$ \\
\hline \multirow{2}{*}{ Elastic properties } & $G_{0}$ & 135 & 135 \\
\hline & $v$ & $0.15 *$ & $0.14 *$ \\
\hline \multirow{5}{*}{ Critical state properties } & $e_{o}$ & 0.773 & 0.812 \\
\hline & $\lambda$ & 0.01 & 0.0189 \\
\hline & $\xi$ & 0.7 & 0.7 \\
\hline & $M$ & 1.24 & 1.296 \\
\hline & $c_{1}$ & 0.7 & 0.7 \\
\hline Yield surface & $m$ & $0.05 *$ & $0.05^{*}$ \\
\hline $\begin{array}{c}\text { Parameter for shape of } \\
\text { plastic potential }\end{array}$ & $c_{2}$ & $0.78^{*}$ & $0.78 *$ \\
\hline \multirow{3}{*}{$\begin{array}{l}\text { Parameters influencing } \\
\text { plastic modulus }\end{array}$} & $h_{\text {in }}$ & 5.1 & 7.5 \\
\hline & $c_{h}$ & 0.96 & 1.01 \\
\hline & $n^{b}$ & 2.1 & 2.0 \\
\hline \multirow{2}{*}{$\begin{array}{l}\text { Parameters influencing } \\
\text { dilatancy }\end{array}$} & $A_{0}$ & 0.6 & 0.84 \\
\hline & $n^{d}$ & 2.5 & 3.4 \\
\hline \multirow{4}{*}{$\begin{array}{c}\text { Anisotropy and state } \\
\text { parameter scaling } \\
\text { parameters }\end{array}$} & $a$ & - & 0.27 \\
\hline & $b_{1}$ & - & 2.5 \\
\hline & $b_{2}$ & - & $-0.833 *$ \\
\hline & $k$ & - & 1.5 \\
\hline
\end{tabular}

* Assumed 
Table 4 : UWA silica sand model parameters obtained using optimisation and from individual tests

\begin{tabular}{|c|c|c|c|c|c|c|}
\hline & $n^{b}$ & $n^{d}$ & $A_{0}$ & $\boldsymbol{h}_{\text {in }}$ & $\boldsymbol{G}_{0}$ & $\begin{array}{c}\text { CSL parameters }(\Gamma, \lambda, \xi, M \text { and } \\
\left.c_{l}\right) \text { and } \boldsymbol{m}, \boldsymbol{c}_{2}, \boldsymbol{v}, \boldsymbol{c}_{\boldsymbol{h}}\end{array}$ \\
\hline Globally optimised set & 2.0 & 3.4 & 0.84 & 7.5 & 135 & Identical as in Table 3 \\
\hline $\begin{array}{c}\text { Test } 1 \text { (drained) } \\
e_{0}=0.66, K_{0}=0.5 \\
\sigma_{v}^{\prime}=1000 \mathrm{kPa}\end{array}$ & 2.1 & 3.9 & 1.1 & 4.9 & 100 & \multirow{6}{*}{ Identical as in Table 3} \\
\hline $\begin{array}{c}\text { Test } 2(\text { drained }) \\
e_{0}=0.679, K_{0}=0.5 \\
\sigma_{v}^{\prime}=100 \mathrm{kPa}\end{array}$ & 1.85 & 2.9 & 0.7 & 4 & 85 & \\
\hline $\begin{array}{c}\text { Test } 3 \text { (drained) } \\
e_{0}=0.717, K_{0}=0.5 \\
\sigma_{v}^{\prime}=40 \mathrm{kPa}\end{array}$ & 2.6 & 2.9 & 0.7 & 5 & 105 & \\
\hline $\begin{array}{c}\text { Test } 4 \text { (drained }) \\
e_{0}=0.7346, K_{0}=1.0 \\
\sigma_{v}{ }^{\prime}=100 \mathrm{kPa}\end{array}$ & 2.7 & 3.7 & 1.05 & 7.8 & 140 & \\
\hline $\begin{array}{c}\text { Mean values } \\
(\% \text { difference from } \\
\text { optimised set }) \\
\end{array}$ & $\begin{array}{r}2.31 \\
(15.5) \\
\end{array}$ & $\begin{array}{r}3.35 \\
(-1.47) \\
\end{array}$ & $\begin{array}{l}0.89 \\
(5.9)\end{array}$ & $\begin{array}{c}5.42 \\
(-27.7)\end{array}$ & $\begin{array}{r}107.5 \\
(-20.4) \\
\end{array}$ & \\
\hline COV (in the mean set) & 0.15 & 0.14 & 0.21 & 0.26 & 0.19 & \\
\hline
\end{tabular}




\section{University Library}

\section{- M M N E R VA A gateway to Melbourne's research publications}

Minerva Access is the Institutional Repository of The University of Melbourne

Author/s:

Roy, A;Chow, SH;O'Loughlin, CD;Randolph, MF;Whyte, S

Title:

Use of a bounding surface model in predicting element tests and capacity in boundary value problems

Date:

2021-06-01

Citation:

Roy, A., Chow, S. H., O'Loughlin, C. D., Randolph, M. F. \& Whyte, S. (2021). Use of a bounding surface model in predicting element tests and capacity in boundary value problems. CANADIAN GEOTECHNICAL JOURNAL, 58 (6), pp.782-799. https:// doi.org/10.1139/cgj-2019-0841.

Persistent Link:

http://hdl.handle.net/11343/251838 